\title{
MILLIHERTZ QUASI-PERIODIC OSCILLATIONS AND THERMONUCLEAR BURSTS FROM TERZAN 5: A SHOWCASE OF BURNING REGIMES
}

\author{
M. Linares ${ }^{1,5}$, D. Altamirano ${ }^{2}$, D. Chakrabarty ${ }^{1}$, A. Cumming ${ }^{3}$, and L. Keek ${ }^{4}$ \\ ${ }^{1}$ Massachusetts Institute of Technology, Kavli Institute for Astrophysics and Space Research, Cambridge, MA 02139, USA \\ 2 Astronomical Institute "Anton Pannekoek," University of Amsterdam and Center for High-Energy Astrophysics, \\ P.O. BOX 94249, 1090 GE Amsterdam, The Netherlands \\ ${ }^{3}$ Department of Physics, McGill University, 3600 Rue University, Montreal, QC H3A 2T8, Canada \\ ${ }^{4}$ School of Physics and Astronomy, University of Minnesota, 116 Church Street SE, Minneapolis, MN 55455, USA \\ Received 2011 November 11; accepted 2012 February 5; published 2012 March 9
}

\begin{abstract}
We present a comprehensive study of the thermonuclear bursts and millihertz quasi-periodic oscillations (mHz QPOs) from the neutron star (NS) transient and $11 \mathrm{~Hz}$ X-ray pulsar IGR J17480-2446, located in the globular cluster Terzan 5. The increase in burst rate that we found during its 2010 outburst, when persistent luminosity rose from 0.1 to 0.5 times the Eddington limit, is in qualitative agreement with thermonuclear burning theory yet contrary to all previous observations of thermonuclear bursts. Thermonuclear bursts gradually evolved into a $\mathrm{mHz}$ QPO when the accretion rate increased, and vice versa. The $\mathrm{mHz}$ QPOs from IGR J17480-2446 resemble those previously observed in other accreting NSs, yet they feature lower frequencies (by a factor $\sim 3$ ) and occur when the persistent luminosity is higher (by a factor 4-25). We find four distinct bursting regimes and a steep (close to inverse cubic) decrease of the burst recurrence time with increasing persistent luminosity. We compare these findings to nuclear burning models and find evidence for a transition between the pure helium and mixed hydrogen/helium ignition regimes when the persistent luminosity was about 0.3 times the Eddington limit. We also point out important discrepancies between the observed bursts and theory, which predicts brighter and less frequent bursts, and suggest that an additional source of heat in the NS envelope is required to reconcile the observed and expected burst properties. We discuss the impact of NS magnetic field and spin on the expected nuclear burning regimes, in the context of this particular pulsar.
\end{abstract}

Key words: accretion, accretion disks - binaries: close - globular clusters: individual (Terzan 5) - stars: neutron X-rays: binaries - X-rays: individuals (IGR J17480-2446)

Online-only material: color figures

\section{INTRODUCTION}

Matter accreted onto neutron stars (NSs) is piled up and compressed, settling toward regions of increasing density and temperature. In this process, depending on the rate at which accretion proceeds, both stable and unstable thermonuclear burning of the accreted $\mathrm{H}$ and $\mathrm{He}$ into heavier elements are expected (Fujimoto et al. 1981). The main parameter thought to determine the different burning regimes is the mass accretion rate on the NS per unit surface area, $\dot{m}$ (e.g., Fujimoto et al. 1981; Bildsten 1998). When the burning layer becomes thermally unstable heat cannot be transported as fast as it is produced and a thermonuclear runaway occurs, producing a shell flash that releases $10^{38}-10^{39} \mathrm{erg}$ in tens of seconds (we do not discuss herein long bursts and superbursts, which are more energetic, $10^{40}-10^{42}$ erg, and much less common; e.g., Keek \& in't Zand 2008). Most of the energy is deposited in the outermost layers of the NS within a few seconds and radiated away thermally for tens of seconds while the photosphere cools down. Type I X-ray bursts, which feature the spectral imprint of such photospheric cooling, were discovered in low-mass X-ray binaries (LMXBs) more than 30 years ago (Grindlay et al. 1976; Belian et al. 1976; Hoffman et al. 1978) and promptly identified as thermonuclear bursts from accreting NSs (Woosley \& Taam 1976; Maraschi \& Cavaliere 1977; Lewin \& Joss 1977). Joss \& Li (1980) pointed out that a strong NS magnetic field can act to stabilize nuclear burning in different ways, which may explain the fact

\footnotetext{
5 Rubicon Fellow.
}

that no thermonuclear bursts have been observed to date from accreting NSs in high-mass X-ray binaries (HMXBs; see further discussion in Section 4.3).

Although direct observational evidence of stable thermonuclear burning on accreting NSs has been elusive, as this is outshined by the much more efficient accretion-powered "persistent" emission, theoretical arguments and indirect observational evidence (Taam 1981; Fujimoto et al. 1981; van Paradijs et al. 1988; Lewin et al. 1993; Bildsten 1998) suggest that at very high $\dot{m}$, close to or above the Eddington mass accretion rate (Sections 2 and 4), thermonuclear burning of the accreted $\mathrm{H}$ and $\mathrm{He}$ proceeds only stably. Near the transition between unstable and stable burning, an oscillatory burning regime was predicted by Paczynski (1983), known as marginally stable burning. Revnivtsev et al. (2001) discovered millihertz quasiperiodic oscillations ( $\mathrm{mHz}$ QPOs) in the X-ray flux of three atoll sources (the sub-class of low-luminosity NS-LMXBs; Hasinger \& van der Klis 1989): 4U 1636-536, 4U 1608-52, and Aql X-1 (see also Strohmayer \& Smith 2011). They attributed this new phenomenon to marginally stable burning on the NS surface. The $\mathrm{mHz}$ QPO frequency in one of these systems has been found to decrease with time until a bright type I X-ray burst occurs (Altamirano et al. 2008). The persistent luminosity at which such $\mathrm{mHz}$ QPOs are observed has remained a puzzle, as it suggests a critical $\dot{m}$ about an order of magnitude lower than the stability boundary predicted by theory (e.g., Heger et al. 2007b).

On 2010 October 10, an X-ray transient in the direction of the globular cluster Terzan 5 was discovered with the International 


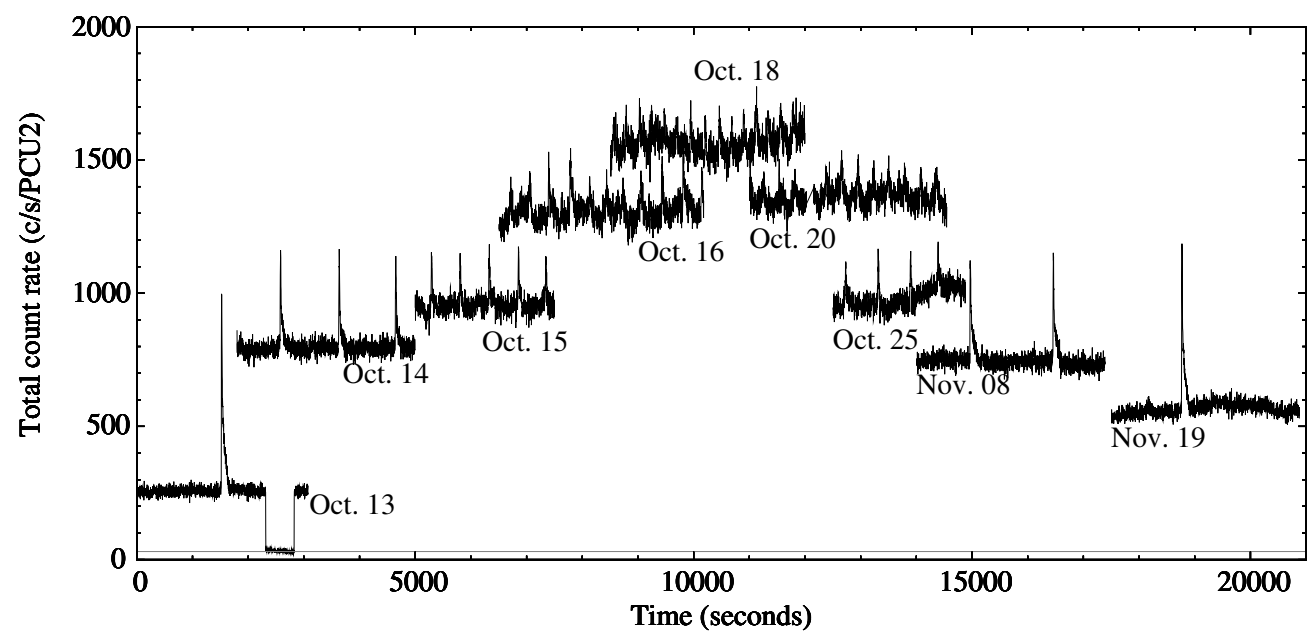

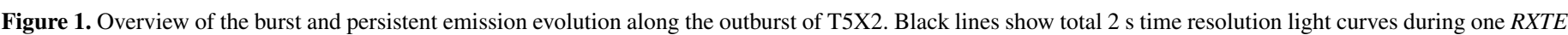

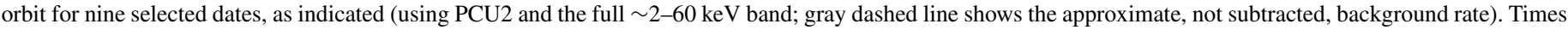

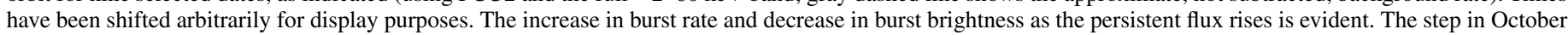
13 was produced by a lunar eclipse (Strohmayer et al. 2010). The source became unobservable for RXTE after November 19 due to solar constraints.

Gamma-Ray Astrophysics Laboratory (Bordas et al. 2010; Chenevez et al. 2010). During the following week, Rossi $X$-ray Timing Explorer (RXTE) observations revealed $11 \mathrm{~Hz}$ pulsations (Strohmayer \& Markwardt 2010) and burst oscillations at the same frequency (Altamirano et al. 2010b; Cavecchi et al. 2011). The Chandra localization (Pooley et al. 2010) confirmed that this was a new NS transient, named IGR J17480-2446 (labeled CX25 or CXOGlb J174804.8-244648 by Heinke et al. 2006). We refer hereinafter to IGR J17480-2446 as T5X2, as this is the second bright X-ray source discovered in Terzan 5 (after EXO 1745-248). A $21.3 \mathrm{hr}$ orbital period was measured from the Doppler shifts on the pulsar frequency (Strohmayer et al. 2010; Papitto et al. 2011), and the NS magnetic field was estimated to be between $10^{8}-10^{10} \mathrm{G}$ based on the inferred magnetospheric radius (Papitto et al. 2011; Miller et al. 2011). This makes T5X2 the type I X-ray burst source (burster) with the slowest known NS spin and with the highest estimates of the NS magnetic field strength. Near the outburst peak T5X2 showed X-ray spectral and variability behavior typical of $Z$ sources (the subclass of high-luminosity NS-LMXBs; Hasinger \& van der Klis 1989), when it was accreting at about half of the Eddington rate (Altamirano et al. 2010a).

Linares et al. (2010a) argued that all the X-ray bursts from T5X2 had a thermonuclear origin, based on the evolution of the burst rate. Given the lack of spectral softening along the tail of many of the bursts and their short recurrence times, Galloway \& in't Zand (2010) suggested that some of the T5X2 bursts were type II instead of type I (i.e., accretioninstead of nuclear-powered). However, the persistent-to-burst energy ratio throughout the October-November outburst of T5X2 was typical of type I X-ray bursts, i.e., fully consistent with the accretion-to-thermonuclear efficiency ratio (Linares et al. 2011; Chakraborty \& Bhattacharyya 2011; Motta et al. 2011). Furthermore, Linares et al. (2011) measured a smooth evolution of the burst luminosity and spectral profiles and put forward a scenario to explain the lack of cooling in the faintest bursts, conclusively identifying all X-ray bursts detected from T5X2 as thermonuclear.

We present a thorough analysis of the $\mathrm{mHz}$ QPOs from T5X2, including but not limited to the ones originally reported by Linares et al. (2010a). We study the $\mathrm{mHz}$ QPO frequency evolution and energy-dependent amplitude, as well as all X-ray bursts from $\mathrm{T} 5 \mathrm{X} 2$ detected with $R X T E$ while the persistent (accretion) luminosity varied along the outburst. Unlike previous studies (Motta et al. 2011; Chakraborty \& Bhattacharyya 2011), we analyze the complete sample of $R X T E$ bursts and compare their properties to theoretical models of thermonuclear burning, along the full range in persistent luminosity ( $\sim 10 \%-50 \%$ of the Eddington luminosity). Section 2 gives the details of the data analysis, and Section 3 presents the main observational results: a smooth evolution between bursts and $\mathrm{mHz}$ QPOs (Figure 1), the $\mathrm{mHz}$ QPO properties in detail and four different bursting regimes during the 2010 October-November outburst of T5X2. In Section 4 we place the unique $\mathrm{mHz}$ QPO and bursting behavior of T5X2 in the framework of thermonuclear burning theory and discuss the possible effects of composition, NS spin, and magnetic field on the observed bursting properties. Section 5 gives our summary and conclusions.

\section{DATA ANALYSIS}

We analyzed all RXTE observations of T5X2 during its 2010 October-December outburst: a total of 46 observations taken between 2010 October 13 and 2010 November 19 (proposal-target number 95437-01). The source became Sun constrained after that date, and was not detected by the Monitor of All-sky X-ray Image on 2010 December 28, indicating that the outburst finished between 2010 November 19 and 2010 December 28. We visually searched for X-ray bursts in the full data set, using 2 s time resolution $2-30 \mathrm{keV}$ light curves. We performed time-resolved spectroscopy of all bursts using high time resolution data (E_125us_64M_0_1s, or GoodXenon when available). We extracted dead-time-corrected spectra in $2 \mathrm{~s}$ time bins, using a $\sim 100 \mathrm{~s}$ long pre- or post-burst interval as background. We added a $1 \%$ systematic error to all channels, grouped them to a minimum of 15 counts per channel when necessary and fitted the resulting spectra within Xspec (ver. 12.6.0q), using a simple blackbody model with the absorbing column density fixed to $1.2 \times 10^{22} \mathrm{~cm}^{-2}$ (Heinke et al. 2006). We used a distance to $\mathrm{T} 5 \mathrm{X} 2$ of $6.3 \mathrm{kpc}$, the highest value reported from Hubble Space Telescope photometry of Terzan 5 (Ortolani et al. 2007), consistent with the distance measurement based on photospheric radius expansion bursts from another burster 
in the same globular cluster (Galloway et al. 2008). We note, however, that recent estimates of this distance range between $4.6 \mathrm{kpc}$ and $8.7 \mathrm{kpc}$ (Cohn et al. 2002; Ortolani et al. 2007; Lanzoni et al. 2010), and therefore any value of the luminosity, energy, and mass accretion rate has a systematic uncertainty of a factor $\sim 3.6$.

We measured the burst rise time ( $t_{\text {rise }}$, as defined in Galloway et al. 2008, i.e., the time to go from $25 \%$ to $90 \%$ of the peak count rate) and the total radiated energy $\left(E_{\mathrm{b}}\right)$ by integrating the bolometric luminosity along each burst. We defined the wait time, $t_{\text {wait }}$, as the time elapsed between the peak of the previous burst and the peak of a given burst, available when no data gaps were present before the burst. We measured the daily-averaged burst recurrence time, $t_{\text {rec }}$, as the total exposure time during one day (excluding those orbits where no bursts are detected) divided by the number of bursts detected on that day. Therefore, the instantaneous and daily-averaged burst rates, $v_{\text {burst }}$, are given by $t_{\text {wait }}^{-1}$ and $t_{\text {rec }}^{-1}$, respectively. When only one burst was detected on a given day, we considered $t_{\mathrm{rec}}$ an approximate lower limit on the recurrence time. We also obtained daily averages of $E_{\mathrm{b}}$, peak burst luminosity $\left(L_{\text {peak }}\right)$, blackbody temperature $\left(k T_{\text {peak }}\right)$, and radius $\left(R_{\text {peak }}\right)$, following the same method described in Linares et al. (2011): peak burst values correspond to a $4 \mathrm{~s}$ long interval around the burst peak.

Due to the smooth evolution from a series of bursts into a $\mathrm{mHz}$ QPO and vice versa (see Figure 1 and Section 3) the distinction between "frequent bursts" and $\mathrm{mHz}$ QPOs is, to some extent, arbitrary. We searched for $\mathrm{mHz}$ QPOs all observations taken when the daily-averaged burst recurrence time was shorter than $350 \mathrm{~s}$, which corresponds to 10 observations between MJDs 55485-55490 (around the outburst peak). Given the typical duration of a continuous RXTE observation segment (an "orbit"), this threshold ensures that about 10 or more QPO cycles, or bursts, are observed without interruption. To study the $\mathrm{mHz}$ QPOs in those RXTE orbits we used 2-60 keV $1 \mathrm{~s}$ bin light curves from all active Proportional Counter Units (PCUs) combined. We then calculated a Lomb-Scargle periodogram (LSP, oversampled by a factor of three; Lomb 1976; Scargle 1982) for each light curve and measured the $\mathrm{mHz} \mathrm{QPO}$ frequency, $v_{\mathrm{QPO}}$, as that frequency with the highest power in the periodogram. The corresponding period was then used to fold the backgroundcorrected light curve (using background rates from pcabackest in $16 \mathrm{~s}$ steps) and produce a $\mathrm{mHz}$ QPO-folded profile, from which we measured the fractional $\mathrm{rms}$ amplitude. To investigate the energy dependence of the $\mathrm{mHz}$ QPO amplitude, we also produced light curves in five different energy bands (in $\mathrm{keV}$ : $2-3,3-5.5,5.5-9.5,9.5-21$, and 21-53), folded the light curve at the period found in the respective $2-60 \mathrm{keV}$ range data set and measured the "rms spectrum" of the $\mathrm{mHz}$ QPOs. We also performed $2048 \mathrm{~s}$ long fast Fourier transforms (FFTs) using the 2-60 keV energy band and the same RXTE orbits, in order to constrain the $\mathrm{mHz}$ QPO coherence or "quality factor."

In order to measure the persistent (accretion) luminosity, we extracted one dead-time-corrected spectrum per observation from Standard 2 data, excluding all bursts and subtracting the background spectrum estimated with the bright source background model and pcabackest (ver. 3.8). We then fitted each persistent spectrum with a model consisting of a disk blackbody, a power law and a Gaussian line with energy fixed at $6.5 \mathrm{keV}$, correcting for absorption as above. We calculated the $2-50 \mathrm{keV}$ persistent luminosity $\left(L_{2}-50\right)$ from the best-fit model. Furthermore, we measured the $0.01-2 \mathrm{keV}$ unabsorbed flux from a simultaneous fit to the T5X2 spectrum measured by the Swift
X-Ray Telescope $(0.5-10 \mathrm{keV})$ and the RXTE Proportional Counter Array (PCA; 2.5-25 keV) on MJD 55501 (2010 November 01), extrapolating the phabs*simpl(bbody + diskbb) best-fit model down to $0.01 \mathrm{keV}$, and found a bolometric correction factor of 1.13. This bolometric correction factor converts $2-50 \mathrm{keV}$ into $0.01-50 \mathrm{keV}$ flux, which we take as bolometric flux given that the persistent spectrum remains soft (photon index 2.4-3.3) throughout the outburst and we therefore do not expect sizable emission above $50 \mathrm{keV}$ (with the only exception of the first observation, when T5X2 was in the hard state and the photon index was $\sim 1.7$ ). Using the same procedure, we found a bolometric correction factor of 1.02 for the $0.5-50 \mathrm{keV}$ luminosity from Cir $\mathrm{X}-1$ reported by Linares et al. (2010b; used in Section 4). We applied these bolometric correction factors in order to estimate the bolometric persistent luminosity: $L_{\text {pers }}$. We use throughout this work an Eddington luminosity of $L_{\text {Edd }}=2.5 \times 10^{38} \mathrm{erg} \mathrm{s}^{-1}$ to calculate Eddington-normalized $L_{\text {pers }}$. To convert from $L_{\text {pers }}$ to $\dot{m}$, we assume homogeneous accretion onto the NS and that the (general-relativistic) gravitational energy of the in-falling matter is fully converted into radiation at the surface of a $1.4 M_{\odot}$ mass, $10 \mathrm{~km}$ radius NS. We define the Eddington mass accretion rate per unit area, $\dot{m}_{\text {Edd }}$, as the mass accretion rate needed to sustain a luminosity equal to $L_{\mathrm{Edd}}$. With this definition $\dot{m}_{\text {Edd }}=1.2 \times 10^{5} \mathrm{~g} \mathrm{~cm}^{-2} \mathrm{~s}^{-1}$.

\section{RESULTS}

We found and analyzed a total of 398 X-ray bursts occurring between 2010 October 13 and 2010 November 19, including the faint and frequent bursts near the peak of the outburst that form the $\mathrm{mHz}$ QPOs (see below). We show in Figure 1 an overview of the joint evolution of persistent emission and burst properties along the T5X2 outburst. Bursts become more frequent and fainter when the persistent luminosity, $L_{\text {pers }}$, increases, and they turn into brighter and more frequent bursts during the outburst decay. Most interestingly, the X-ray bursts gradually develop into the observed $\mathrm{mHz}$ QPO during the outburst rise, and the $\mathrm{mHz}$ QPO mutates into a series of bursts along the outburst decay (Figure 1), an unprecedented phenomenon among thermonuclear bursters. This also makes T5X2 the most prolific source of thermonuclear bursts known to date (an average burst rate over more than a month of $4.9 \mathrm{hr}^{-1}$; $5.5 \mathrm{hr}^{-1}$ excluding orbits where no bursts were detected), with the shortest recurrence times between thermonuclear bursts observed to date (as short as 200 s; cf. Linares et al. 2009; Keek et al. 2010).

The smooth metamorphosis between bursts and $\mathrm{mHz}$ QPOs can be seen qualitatively in Figure 1, and quantitatively by studying the evolution of several burst properties (Figure 2 and Table 1). Burst rate, rise time, and duration as well as peak burst luminosity, $L_{\text {peak }}$, and total radiated energy, $E_{\mathrm{b}}$ (see also Linares et al. 2011); all evolve gradually while $L_{\text {pers }}$ changes by a factor of $\sim 5$ along the outburst. Figure 2 (middle panel) also shows that the burst rate equals the $\mathrm{mHz}$ QPO frequency during the outburst peak, $v_{\text {burst }}=v_{\mathrm{QPO}}$, as expected given that the $\mathrm{mHz}$ QPOs are simply formed by a series of faint and frequent bursts. This unique behavior makes the distinction between bursts and $\mathrm{mHz}$ QPOs somewhat arbitrary. As explained in Section 2, our practical definition of $\mathrm{mHz}$ QPO requires burst recurrence times shorter than $350 \mathrm{~s}$, so that typically 10 or more QPO cycles are observed without interruption. On a few occasions we find that one burst is missing from the series of regular bursts, and the 


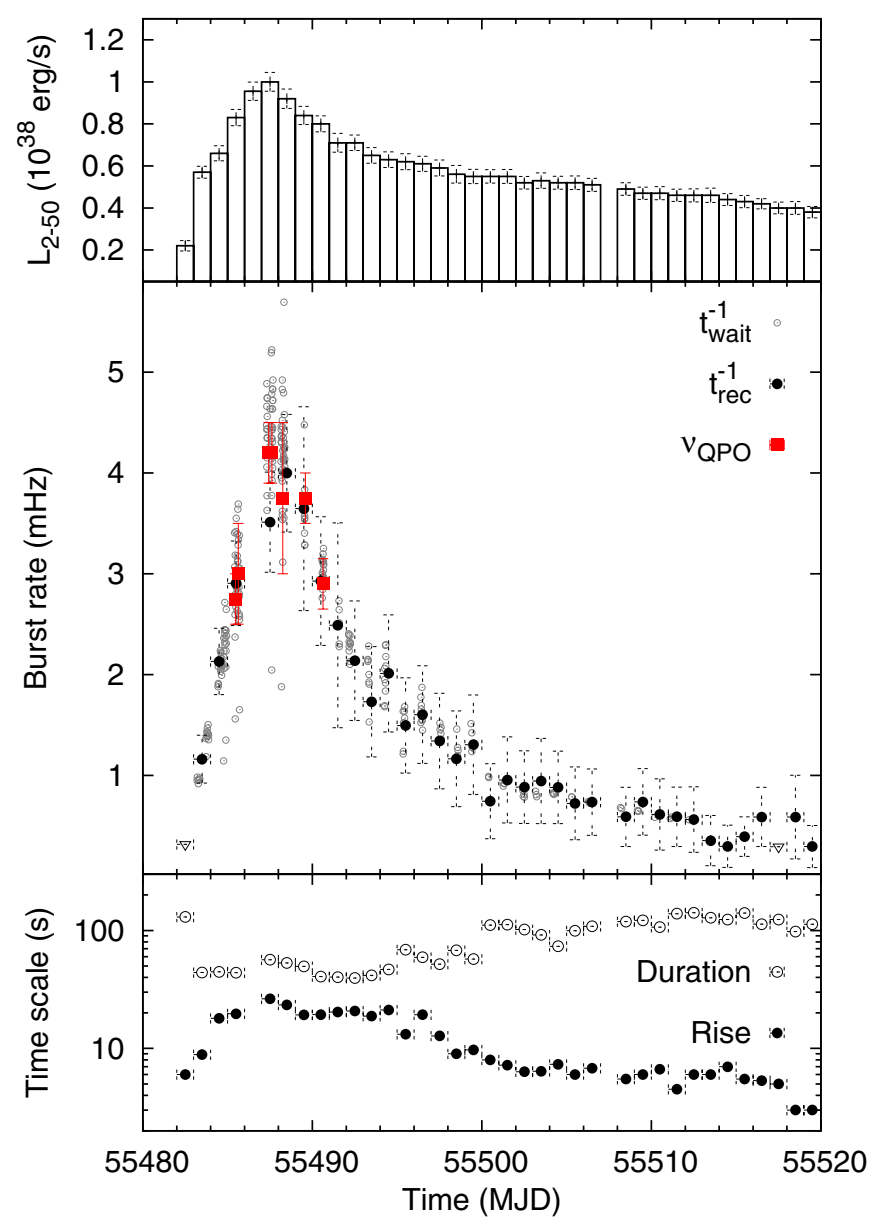

Figure 2. From top to bottom panels, evolution along the outburst of (1) persistent 2-50 keV luminosity; (2) burst rate as measured from $t_{\text {wait }}$ (open gray circles), $t_{\text {rec }}$ (filled black circles), and $\mathrm{mHz}$ QPO frequency (red filled squares; see Section 2 for definitions); and (3) burst rise time and duration. Gray and black circles show individual burst measurements and daily averages, respectively. Open triangles show burst rate daily averages based on one single burst, which we consider as upper limits.

(A color version of this figure is available in the online journal.)

corresponding values of $v_{\text {burst }}=t_{\text {wait }}^{-1}$ for individual bursts are a factor $\sim 2$ lower than the general trend, as can be seen in Figure 2 (middle panel). Interestingly, a similar behavior with sporadic "missing bursts" is seen in the $\mathrm{mHz}$ QPO simulations presented in Heger et al. (2007b).

\section{1. $\mathrm{mHz} Q P O s$}

We report the discovery of several instances of $\mathrm{mHz}$ QPOs during the peak of the T5X2 outburst, on 2010 October 16, 18, 19, 20, and 21 (MJDs 55485-55490; Linares et al. 2010a for the initial report of $\mathrm{mHz}$ QPOs on 2010 October 18 and 19). Two examples of $\mathrm{mHz}$ QPO light curves are shown in Figure 3, each spanning one RXTE orbit. Table 2 shows the main QPO properties: fractional rms amplitudes between $1.3 \%$ and $2.2 \%$ (in the 2-60 keV band) and $v_{\mathrm{QPO}}$ in the range $2.8-4.2 \mathrm{mHz}$ (note that the lower end of this frequency range corresponds to our $\mathrm{mHz}$ QPO definition; Sections 2 and 3). Figure 4 presents LSPs of two cases, on October 16 and 18, clearly showing the change in $v_{\mathrm{QPO}}$ as well as the harmonic structure (up to four overtones are visible in the LSPs). By inspecting the power spectra obtained from 2048 s long FFTs, we find that the $\mathrm{mHz}$ QPO power is in all cases spread over one or two frequency bins, from which we derive a limit on the FWHM $\lesssim 1 \mathrm{mHz}$. For the $2.8-4.2 \mathrm{mHz}$ QPO frequencies this corresponds to a lower limit on the quality factor $\left(Q \equiv v_{\mathrm{QPO}} / \mathrm{FWHM}\right)$ of $Q \gtrsim 3$, which reveals a fairly coherent QPO (a fairly constant $t_{\text {wait }}$ ).

We present in Figure 5 light curves folded at the $\mathrm{mHz}$ QPO period, showing folded burst/QPO profiles for the same two dates, October 16 and 18. In both cases the burst/QPO profile is peaked and highly non-sinusoidal (as seen also in the raw, unfolded light curves; Figure 3), which explains the harmonic content. On October 18, when $v_{\mathrm{QPO}}$ was highest (Table 2), we find a nearly symmetric burst/QPO profile. On October 16 the burst/QPO profile is slightly asymmetric, with the rise faster than the decay. We measure a $\mathrm{mHz}$ QPO fractional rms amplitude in the range $1.3 \%-2.2 \%$, in the total ( 2-60 keV) PCA band. Furthermore, from our measurements of the $\mathrm{mHz}$ QPO amplitude at different energies (Section 2 and Figure 6) we find that its fractional rms amplitude increases between $\sim 2$ and $\sim 20 \mathrm{keV}$, from $\sim 1.4 \%$ to $\sim 2.8 \%$ in the two cases presented in Figure 6. At energies higher than $20 \mathrm{keV}$ the $\mathrm{mHz}$ QPO is not visible by the naked eye in the light curves, nor is it conclusively detected using LSPs, FFTs, or folded light curves. The presence of red noise with variable strength gives rise to different upper limits on the fractional rms amplitude of the $\mathrm{mHz}$ QPO above $20 \mathrm{keV}$ (between 1.4\% and 3\%; Figure 6). As discussed in Section 4.2, such an increase in QPO amplitude up to $20 \mathrm{keV}$ is in contrast with $\mathrm{mHz}$ QPOs from other bursting NSs, which showed a fractional amplitude decreasing with increasing energy between 2 and $5 \mathrm{keV}$ (Revnivtsev et al. 2001).

\subsection{Burst Properties versus Accretion Rate}

We present in this section the relation between burst properties and $\dot{m}$, as inferred from $L_{\text {pers }}$, including the faintest and most frequent bursts which form the $\mathrm{mHz}$ QPOs (see above). Figure 2 shows $L_{\text {peak }}, E_{\mathrm{b}}$, and $t_{\text {rec }}$ as a function of the Eddingtonnormalized $L_{\text {pers }}$ and $\dot{m}$ (Section 2 ). The overall trend is that of an anticorrelation between $L_{\text {peak }}, E_{\mathrm{b}}$, and $t_{\text {rec }}$ on the one hand and $L_{\text {pers }}$ on the other (see also Figure 1 in Linares et al. 2011; Motta et al. 2011). Closer inspection of the burst properties over the full $L_{\text {pers }}$ range $\left(0.1-0.5 L_{\text {Edd }}\right.$; see Figure 7$)$ reveals a more complex and interesting behavior, namely, four different bursting regimes.

1. At the lowest persistent luminosities, $0.1<L_{\text {pers }} / L_{\text {Edd }}<$ 0.2 , when $L_{\text {pers }}$ increases both $L_{\text {peak }}$ and $E_{\mathrm{b}}$ (and possibly $t_{\text {rec }}$ ) decrease moderately, from $L_{\text {peak }} \simeq 4.6 \times 10^{37} \mathrm{erg} \mathrm{s}^{-1}$, $E_{\mathrm{b}} \simeq 1.6 \times 10^{39} \mathrm{erg}$ to $L_{\text {peak }} \simeq 3.7 \times 10^{37} \mathrm{erg} \mathrm{s}^{-1}$, $E_{\mathrm{b}} \simeq 1.3 \times 10^{39} \mathrm{erg}$. We refer to this $0.1-0.2 L_{\text {Edd }}$ regime as slow decrease, or regime A (see Figure 7).

2. At higher persistent luminosities, $0.2<L_{\text {pers }} / L_{\text {Edd }}<0.3$, we find $L_{\text {peak }}$ and $E_{\mathrm{b}}$ to be steeply anticorrelated with $L_{\text {pers }}$, while $t_{\text {rec }}$ also decreases from $200 \mathrm{~s}$ to $400 \mathrm{~s}$ with increasing $L_{\text {pers }}$. We refer to this $0.2-0.3 L_{\text {Edd }}$ regime as fast drop, or regime $B$ (see Figure 7 ).

3. At the highest persistent luminosities ( $\left.L_{\text {pers }}>0.3 L_{\text {Edd }}\right)$, when $\mathrm{mHz}$ QPOs are detected, burst peak luminosity and energy reach approximately constant values (saturation, or regime $C$ ): $L_{\text {peak }} \simeq 1.2 \times 10^{37} \mathrm{erg} \mathrm{s}^{-1}$ and $E_{\mathrm{b}} \simeq$ $0.3 \times 10^{39} \mathrm{erg}$. The burst recurrence time, however, keeps decreasing with increasing $L_{\text {pers }}$ from $t_{\text {rec }} \simeq 400 \mathrm{~s}$ down to $\sim 240$ s on 2010 October 18 (Figure 7 and Table 2).

4. Finally, during incursions into the flaring/normal branches near the outburst peak, when T5X2 showed $Z$ source 
Table 1

Daily-averaged Burst Properties and Persistent Luminosity from T5X2

\begin{tabular}{|c|c|c|c|c|c|c|c|c|}
\hline $\begin{array}{l}\text { Date }^{\mathrm{a}} \\
(\mathrm{MJD})\end{array}$ & $\begin{array}{c}\text { Exposure }^{b} \\
\text { (s) }\end{array}$ & Bursts $^{c}$ & $\begin{array}{l}t_{\mathrm{rec}}^{\mathrm{d}} \\
(\mathrm{s})\end{array}$ & $\begin{array}{c}E_{\mathrm{b}} \mathrm{e}^{\mathrm{e}} \\
\left(10^{39} \mathrm{erg}\right)\end{array}$ & $\begin{array}{c}L_{\text {peak }}{ }^{\mathrm{e}} \\
\left(10^{37} \text { erg s }^{-1}\right)\end{array}$ & $\begin{array}{c}k T_{\text {peak }}{ }^{\mathrm{f}} \\
(\mathrm{keV})\end{array}$ & $\begin{array}{c}R_{\text {peak }} \mathrm{e} \\
(\mathrm{km})\end{array}$ & $\begin{array}{c}L_{2-50}{ }^{\mathrm{e}} \\
\left(10^{37} \text { erg s}^{-1}\right)\end{array}$ \\
\hline 55482 & 3158 & 1 & 3158.0 & $1.60 \pm 0.07$ & $4.60 \pm 0.57$ & $2.27 \pm 0.08$ & $3.67 \pm 0.23$ & $2.20 \pm 0.25$ \\
\hline 55483 & 20666 & 24 & 861.1 & $0.42 \pm 0.01$ & $2.00 \pm 0.10$ & $2.13 \pm 0.03$ & $2.70 \pm 0.07$ & $5.70 \pm 0.28$ \\
\hline 55484 & 19709 & 42 & 469.3 & $0.28 \pm 0.01$ & $1.30 \pm 0.07$ & $2.01 \pm 0.03$ & $2.35 \pm 0.07$ & $6.60 \pm 0.36$ \\
\hline 55485 & 16514 & 48 & 344.0 & $0.29 \pm 0.01$ & $1.30 \pm 0.07$ & $1.91 \pm 0.02$ & $2.69 \pm 0.07$ & $8.30 \pm 0.39$ \\
\hline 55486 & [17334] & {$[0]$} & $\ldots$ & $\cdots$ & $\ldots$ & $\ldots$ & $\ldots$ & $9.55 \pm 0.44$ \\
\hline 55487 & 14235 [6980] & $50[0]$ & 284.7 & $0.36 \pm 0.01$ & $1.30 \pm 0.08$ & $1.72 \pm 0.02$ & $3.01 \pm 0.10$ & $10.00 \pm 0.45$ \\
\hline 55488 & 11755 [3633] & $47[0]$ & 250.1 & $0.37 \pm 0.01$ & $1.20 \pm 0.07$ & $1.69 \pm 0.02$ & $3.13 \pm 0.09$ & $9.20 \pm 0.46$ \\
\hline 55489 & 3565 [3752] & $13[0]$ & 274.2 & $0.31 \pm 0.01$ & $1.00 \pm 0.11$ & $1.77 \pm 0.05$ & $2.48 \pm 0.14$ & $8.40 \pm 0.43$ \\
\hline 55490 & 7172 & 21 & 341.5 & $0.28 \pm 0.01$ & $1.10 \pm 0.09$ & $1.82 \pm 0.03$ & $2.65 \pm 0.10$ & $8.00 \pm 0.38$ \\
\hline 55491 & 2410 & 6 & 401.7 & $0.23 \pm 0.01$ & $0.99 \pm 0.16$ & $1.60 \pm 0.06$ & $3.21 \pm 0.26$ & $7.10 \pm 0.45$ \\
\hline 55492 & 6079 & 13 & 467.6 & $0.30 \pm 0.01$ & $1.30 \pm 0.14$ & $1.80 \pm 0.05$ & $2.75 \pm 0.16$ & $7.10 \pm 0.37$ \\
\hline 55493 & 5780 & 10 & 578.0 & $0.24 \pm 0.01$ & $1.10 \pm 0.13$ & $1.59 \pm 0.04$ & $3.28 \pm 0.20$ & $6.50 \pm 0.37$ \\
\hline 55494 & 5963 & 12 & 496.9 & $0.27 \pm 0.01$ & $1.20 \pm 0.14$ & $1.73 \pm 0.05$ & $2.95 \pm 0.18$ & $6.30 \pm 0.37$ \\
\hline 55495 & 6685 & 10 & 668.5 & $0.51 \pm 0.01$ & $1.70 \pm 0.11$ & $1.90 \pm 0.03$ & $3.10 \pm 0.11$ & $6.20 \pm 0.38$ \\
\hline 55496 & 6860 & 11 & 623.6 & $0.36 \pm 0.01$ & $1.30 \pm 0.11$ & $1.83 \pm 0.04$ & $2.79 \pm 0.12$ & $6.10 \pm 0.36$ \\
\hline 55497 & 5964 & 8 & 745.5 & $0.37 \pm 0.02$ & $1.60 \pm 0.15$ & $1.97 \pm 0.04$ & $2.76 \pm 0.13$ & $5.90 \pm 0.38$ \\
\hline 55498 & 5150 & 6 & 858.3 & $0.48 \pm 0.02$ & $1.60 \pm 0.14$ & $1.94 \pm 0.04$ & $2.95 \pm 0.13$ & $5.60 \pm 0.42$ \\
\hline 55499 & 5364 & 7 & 766.3 & $0.35 \pm 0.01$ & $1.60 \pm 0.13$ & $1.96 \pm 0.04$ & $2.81 \pm 0.11$ & $5.50 \pm 0.34$ \\
\hline 55500 & 5372 & 4 & 1343.0 & $0.74 \pm 0.03$ & $1.90 \pm 0.17$ & $1.98 \pm 0.04$ & $3.11 \pm 0.14$ & $5.50 \pm 0.32$ \\
\hline 55501 & 5236 & 5 & 1047.2 & $0.79 \pm 0.02$ & $2.10 \pm 0.16$ & $2.02 \pm 0.04$ & $3.07 \pm 0.11$ & $5.50 \pm 0.33$ \\
\hline 55502 & 6789 & 6 & 1131.5 & $0.87 \pm 0.02$ & $2.50 \pm 0.15$ & $2.06 \pm 0.03$ & $3.19 \pm 0.10$ & $5.20 \pm 0.30$ \\
\hline 55503 & 5292 & 5 & 1058.4 & $0.74 \pm 0.02$ & $2.30 \pm 0.16$ & $2.11 \pm 0.04$ & $3.00 \pm 0.10$ & $5.30 \pm 0.37$ \\
\hline 55504 & 6810 & 6 & 1135.0 & $0.57 \pm 0.02$ & $2.10 \pm 0.14$ & $2.15 \pm 0.04$ & $2.75 \pm 0.09$ & $5.20 \pm 0.31$ \\
\hline 55505 & 5536 & 4 & 1384.0 & $0.74 \pm 0.02$ & $2.20 \pm 0.18$ & $2.12 \pm 0.04$ & $2.88 \pm 0.12$ & $5.20 \pm 0.32$ \\
\hline 55506 & 6803 & 5 & 1360.6 & $0.93 \pm 0.02$ & $2.60 \pm 0.17$ & $2.14 \pm 0.04$ & $3.06 \pm 0.10$ & $5.10 \pm 0.31$ \\
\hline 55508 & 6799 & 4 & 1699.8 & $1.00 \pm 0.03$ & $2.70 \pm 0.19$ & $2.18 \pm 0.04$ & $3.07 \pm 0.10$ & $4.90 \pm 0.30$ \\
\hline 55509 & 6781 & 5 & 1356.2 & $1.06 \pm 0.02$ & $2.90 \pm 0.16$ & $2.21 \pm 0.03$ & $3.08 \pm 0.08$ & $4.70 \pm 0.29$ \\
\hline 55510 & 4893 & 3 & 1631.0 & $1.02 \pm 0.03$ & $3.10 \pm 0.23$ & $2.08 \pm 0.04$ & $3.54 \pm 0.13$ & $4.70 \pm 0.31$ \\
\hline 55511 & 6788 & 4 & 1697.0 & $1.26 \pm 0.03$ & $3.10 \pm 0.20$ & $2.07 \pm 0.03$ & $3.60 \pm 0.11$ & $4.60 \pm 0.29$ \\
\hline 55512 & 5329 & 3 & 1776.3 & $1.28 \pm 0.03$ & $3.30 \pm 0.21$ & $2.17 \pm 0.04$ & $3.35 \pm 0.11$ & $4.60 \pm 0.31$ \\
\hline 55513 & 5661 & 2 & 2830.5 & $1.37 \pm 0.05$ & $3.30 \pm 0.37$ & $2.30 \pm 0.07$ & $3.01 \pm 0.17$ & $4.60 \pm 0.34$ \\
\hline 55514 & 6737 & 2 & 3368.5 & $1.46 \pm 0.03$ & $3.70 \pm 0.26$ & $2.25 \pm 0.04$ & $3.32 \pm 0.12$ & $4.40 \pm 0.28$ \\
\hline 55515 & 10197 & 4 & 2549.2 & $1.33 \pm 0.02$ & $3.70 \pm 0.18$ & $2.23 \pm 0.03$ & $3.40 \pm 0.08$ & $4.30 \pm 0.29$ \\
\hline 55516 & 6803 & 4 & 1700.8 & $1.37 \pm 0.03$ & $3.70 \pm 0.25$ & $2.16 \pm 0.04$ & $3.61 \pm 0.12$ & $4.20 \pm 0.24$ \\
\hline 55517 & 3410 & 1 & 3410.0 & $1.42 \pm 0.04$ & $3.70 \pm 0.29$ & $2.21 \pm 0.04$ & $3.47 \pm 0.13$ & $4.00 \pm 0.28$ \\
\hline 55518 & 3407 & 2 & 1703.5 & $1.22 \pm 0.03$ & $3.80 \pm 0.26$ & $2.31 \pm 0.04$ & $3.24 \pm 0.11$ & $4.00 \pm 0.30$ \\
\hline 55519 & 6788 & 2 & 3394.0 & $1.45 \pm 0.04$ & $3.90 \pm 0.34$ & $2.19 \pm 0.05$ & $3.61 \pm 0.16$ & $3.80 \pm 0.27$ \\
\hline
\end{tabular}

Notes.

a MJD 55482 is 2010 October 13 and MJD 55519 is 2010 November 19.

${ }^{\mathrm{b}}$ Total daily exposure time in data segments (orbits) where bursts are detected. The total daily exposure time for segments without detected bursts is indicated between square brackets (whenever this time is larger than the burst recurrence time). Thus square brackets indicate periods of intrinsic burst cessation.

c Total number of bursts detected per day. Square brackets indicate periods of intrinsic burst cessation, when no bursts were detected despite long enough exposure time.

d Daily-averaged burst recurrence time.

e Bolometric-integrated burst energy $\left(E_{\mathrm{b}}\right)$ and peak burst luminosity ( $\left.L_{\text {peak }}\right)$. Persistent luminosity $\left(L_{2}-50\right)$ in the $2-50 \mathrm{keV}$ energy band (see Section 2 for bolometric correction). Peak burst blackbody radius $\left(R_{\text {peak }}\right)$ not color- or redshift-corrected. All use a distance of $6.3 \mathrm{kpc}$.

f Peak burst blackbody temperature.

behavior (Altamirano et al. 2010a), neither bursts nor $\mathrm{mHz}$ QPOs were detected. These few episodes without bursting activity, which we refer to as regime $D$, were associated with moderate ( $\sim 30 \%$ ) short (hour-long) drops in $L_{\text {pers }}$ and occurred between MJDs 55486 and 55496 (while $L_{\text {pers }} \gtrsim 0.3 L_{\text {Edd }}$ ). They are noted with square brackets in Table 1.

Interestingly, regimes $\mathrm{C}$ and $\mathrm{D}$ show similar $L_{\text {pers }}(0.3-0.5$ $L_{\text {Edd }}$ ), but their variability and spectral properties differ (i.e., they constitute different "accretion states"; see D. Altamirano et al. 2012, in preparation). We summarize the main bursting properties of all four regimes in Table 4.
In order to quantify the anticorrelations explained above and to characterize the bursting regimes present in $\mathrm{T} 5 \mathrm{X} 2$, we fit the $L_{\text {peak }}, E_{\mathrm{b}}, t_{\text {rec }}$ versus $L_{\text {pers }}$ relations with broken powerlaw functions. The results are shown in Figure 8 and Table 3, and confirm that the two "breaks" or transitions occur at $L_{\text {pers }} \simeq 0.2 L_{\text {Edd }}$ and $L_{\text {pers }} \simeq 0.3 L_{\text {Edd. }}$. Due to the large scatter some of the fits are statistically poor, yet they allow us to constrain the slope and transition luminosity of the three bursting regimes (Figure 8). Remarkably, the $t_{\text {rec }}-L_{\text {pers }}$ relation that we find in regime $\mathrm{B}\left(L_{\text {pers }} \sim 0.2-0.3 L_{\mathrm{Edd}}\right)$ is far from linear, as would be expected from a $t_{\text {rec }} \propto \dot{m}^{-1}$ relation if $\dot{m} \propto L_{\text {pers }}$ (see Section 4.1 for further discussion). Instead, it is close to $t_{\text {rec }}$ $\propto L_{\text {pers }}^{-3}$ : we measure a $t_{\text {rec }}-L_{\text {pers }}$ power-law index in regime 


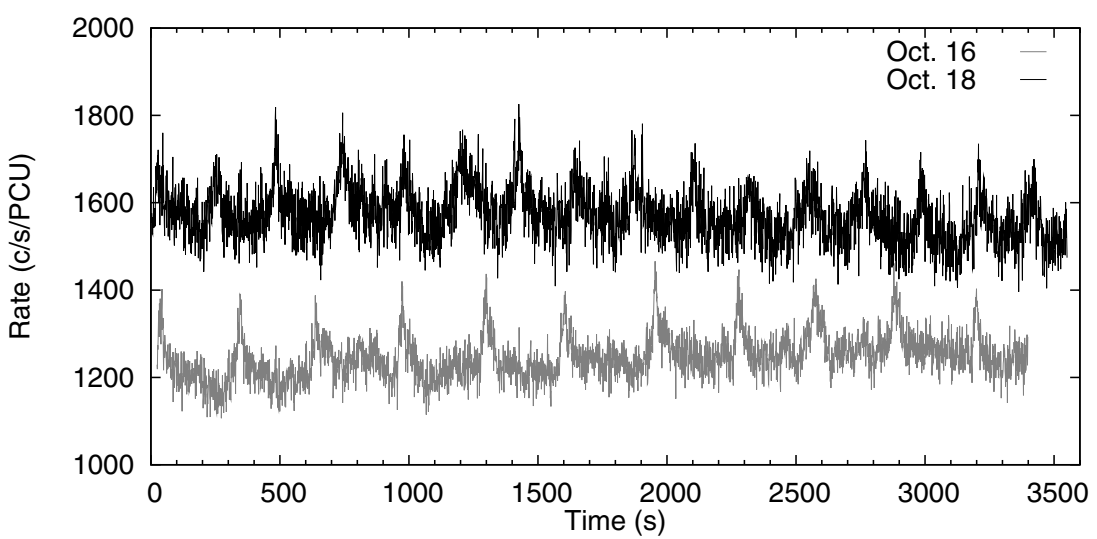

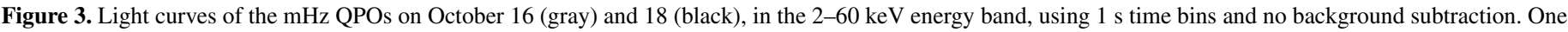

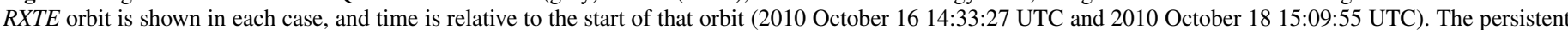

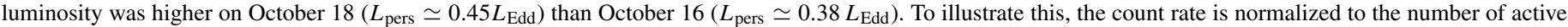
PCUs.

Table 2

Properties of the $\mathrm{mHz}$ QPOs from T5X2

\begin{tabular}{llrcrc}
\hline \hline $\begin{array}{l}\text { Date } \\
\text { (MJD) }\end{array}$ & \multicolumn{1}{c}{ ObsID $^{\mathrm{a}}$} & \multicolumn{1}{c}{$\begin{array}{c}v_{\mathrm{QPO}}{ }^{\mathrm{b}} \\
(\mathrm{mHz})\end{array}$} & \multicolumn{1}{c}{$\begin{array}{c}\mathrm{rms}_{\mathrm{QPO}} \\
(\%)\end{array}$} & $\begin{array}{c}L_{\text {pers }} / 10^{37} \\
\left(\mathrm{erg} \mathrm{s}^{-1}\right)\end{array}$ & $\begin{array}{c}\dot{m} / 10^{4} \\
\left(\mathrm{~g} \mathrm{~cm}^{-2} \mathrm{~s}^{-1}\right)\end{array}$ \\
\hline 55485.46 & $04-00[1]$ & $2.75 \pm 0.2$ & $1.9 \pm 0.1$ & $9.1 \pm 0.4$ & $4.5 \pm 0.2$ \\
55485.63 & $04-01[1,2,3]$ & $3 \pm 0.5$ & $2.2 \pm 0.1$ & $9.9 \pm 0.4$ & $4.9 \pm 0.2$ \\
55487.43 & $06-000[1,4]$ & $4.2 \pm 0.2$ & $1.3 \pm 0.1$ & $11.4 \pm 0.4$ & $5.6 \pm 0.2$ \\
55487.62 & $06-00[1,2]$ & $4.2 \pm 0.2$ & $1.7 \pm 0.2$ & $12.1 \pm 0.5$ & $6.0 \pm 0.2$ \\
55488.26 & $07-00[1]$ & $4.0 \pm 0.5$ & $1.4 \pm 0.1$ & $12.0 \pm 0.5$ & $5.9 \pm 0.2$ \\
55489.59 & $08-00[1]$ & $3.75 \pm 0.2$ & $2.1 \pm 0.1$ & $9.6 \pm 0.5$ & $4.8 \pm 0.2$ \\
55490.63 & $09-00[1,2]$ & $2.9 \pm 0.2$ & $2.2 \pm 0.1$ & $9.1 \pm 0.4$ & $4.5 \pm 0.2$ \\
& & & & &
\end{tabular}

Notes.

a Observation (from proposal-target 95437-01) where the $\mathrm{mHz}$ QPOs are detected, orbit numbers used indicated between square brackets.

b For comparison, the reported values for the "low- $L_{\text {pers }} \mathrm{mHz}$ QPOs" are in the following ranges: $v_{\mathrm{QPO}}=[7-14.3] \mathrm{mHz} ; \mathrm{rms}_{\mathrm{QPO}}=[0.7-1.9] \%(2-5 \mathrm{keV})$; $L_{\text {pers }}=[0.6-3.5] \times 10^{37} \mathrm{erg} \mathrm{s}^{-1}$ (Section 4.2; Revnivtsev et al. 2001; Altamirano et al. 2008).

B of $-3.2 \pm 0.5$ (see further discussion in Section 4 ). We also calculate the mean alpha parameter $\left(\alpha \equiv L_{\text {pers }} \times t_{\text {rec }} / E_{\mathrm{b}}\right)$ from the daily-averaged values reported by Linares et al. (2011) and, after applying the bolometric correction we obtained from broadband X-ray spectral fits (Section 2), we find a mean $\alpha$ of 102 , with a standard deviation of 27 . Figure 9 shows that $\alpha$ increases from $\sim 60$ to $\sim 120$ with increasing $L_{\text {pers }}$ until $L_{\text {pers }} \simeq 0.3 L_{\text {Edd }}$ (regimes $\mathrm{A}$ and B), and decreases at higher luminosities (regime C) to again reach values close to 60 . Figure 9 also shows the burst duration and rise time as a function of $L_{\text {pers }}$ : the general trend is for bursts to become shorter and have slower rise when $L_{\text {pers }}$ increases.

\section{DISCUSSION}

The bursting behavior of T5X2 strikes us as surprising for several reasons. During the last three decades bursts at persistent luminosities $L_{\text {pers }} \gtrsim 0.2 L_{\text {Edd }}$ had proven exceptional and extremely difficult to detect, even when studying a large sample of bursters (e.g., Cornelisse et al. 2003; Galloway et al. 2008, and references therein). This decrease of burst rate at high $L_{\text {pers }}$, opposite to what standard burst theory predicts (Fujimoto et al. 1981; Bildsten 1998), has been attributed in the literature to several effects, including: (1) stable thermonuclear burning becoming more important at high $\dot{m}$ and consuming
Table 3

Broken Power-law Fits to the Burst Properties versus Persistent Luminosity Relations

\begin{tabular}{lccc}
\hline \hline $\begin{array}{l}\text { Relation } \\
\text { (range) }\end{array}$ & $\begin{array}{c}L_{\text {peak }}-L_{\text {pers }} \\
(0.1-0.3)\end{array}$ & $\begin{array}{c}E_{\mathrm{b}}-L_{\text {pers }} \\
(0.1-0.3)\end{array}$ & $\begin{array}{c}t_{\text {rec }}-L_{\text {pers }} \\
(0.17-0.45)\end{array}$ \\
\hline$K^{\mathrm{b}}$ & $2.2 \pm 0.7$ & $0.9 \pm 0.1$ & $10 \pm 7$ \\
$i_{1}$ & $-0.3 \pm 0.2$ & $-0.2 \pm 0.1$ & $-3.2 \pm 0.5$ \\
$L_{\text {break }}\left(L_{\text {Edd }}\right)$ & $0.20 \pm 0.01$ & $0.20 \pm 0.01$ & $0.33 \pm 0.04$ \\
$i_{2}$ & $-2.7 \pm 0.1$ & $-4.4 \pm 0.1$ & $-1.0 \pm 0.9$ \\
$\chi^{2} /$ dof & $38.5 / 25$ & $930 / 25$ & $6.1 / 30$ \\
\hline
\end{tabular}

Notes.

a Pairs of variables fitted with a broken power law: $L_{\text {peak }}, E_{\mathrm{b}}$, and $t_{\text {rec }}$ vs. $L_{\text {pers }}$ (see Figures 7 and 8 ). The $L_{\text {pers }}$ range used in each fit is quoted between brackets, in units of $L_{\mathrm{Edd}} . i_{1}$ and $i_{2}$ represent, respectively, the power-law indices before and after the break (at $L_{\text {break }}$ ).

b Power-law normalization before the break, same units as $L_{\text {peak }}\left(10^{37} \mathrm{erg} \mathrm{s}^{-1}\right)$, $E_{\mathrm{b}}\left(10^{39} \mathrm{erg}\right)$, and $t_{\mathrm{rec}}(\mathrm{s})$.

an increasing fraction of the accreted fuel (van Paradijs et al. 1988), (2) deflagration fronts or "flames" propagating on the NS surface and consuming part of the fuel (Bildsten 1995), and (3) non-spherical accretion confined to a fraction of the NS surface that would increase with $L_{\text {pers }}$ (Bildsten 2000). The large number of bursts observed from T5X2 in 2010 October-November (at an average burst rate over more than a month of $4.9 \mathrm{hr}^{-1}$, Section 3) make T5X2 the most prolific source of thermonuclear bursts known to date. Notably, such copious burst activity was observed when $L_{\text {pers }}$ was in the range $0.1-0.5 L_{\text {Edd }}$, a regime where burst rate is found to decrease drastically in all other bursters. The only source that has shown (albeit sporadically) such high burst rates at similar $L_{\text {pers }}$ is Cir X-1: on 2010 May it featured burst recurrence times as short as 1000-2000 s, when $L_{\text {pers }}$ was $\sim 0.2 L_{\text {Edd }}$ (Linares et al. 2010b). Two other wellknown sources of "high- $\dot{m}$ " bursts, GX 17+2 and Cyg X-2, have shown bursts much less frequently (the mean burst rate over more than 10 years was 0.05 and $0.1 \mathrm{hr}^{-1}$, respectively; Galloway et al. 2008) and when $L_{\text {pers }}$ was close to $L_{\text {Edd }}$. Other systems have shown thermonuclear bursts at intermediate accretion rates $\left(L_{\text {pers }} \simeq 0.1-0.5 L_{\text {Edd }}\right.$; including GX $3+1$, GX 13+1, Ser X-1, 4U 1636-53, 4U 1735-44, 4U 1746-37), but only sporadically ( $t_{\text {wait }}$ of at least $1 \mathrm{hr}$ and typically much longer; Galloway et al. 2008, and references therein). T5X2 is therefore exceptional in that it follows the expected burst rate at high $\dot{m}$ 


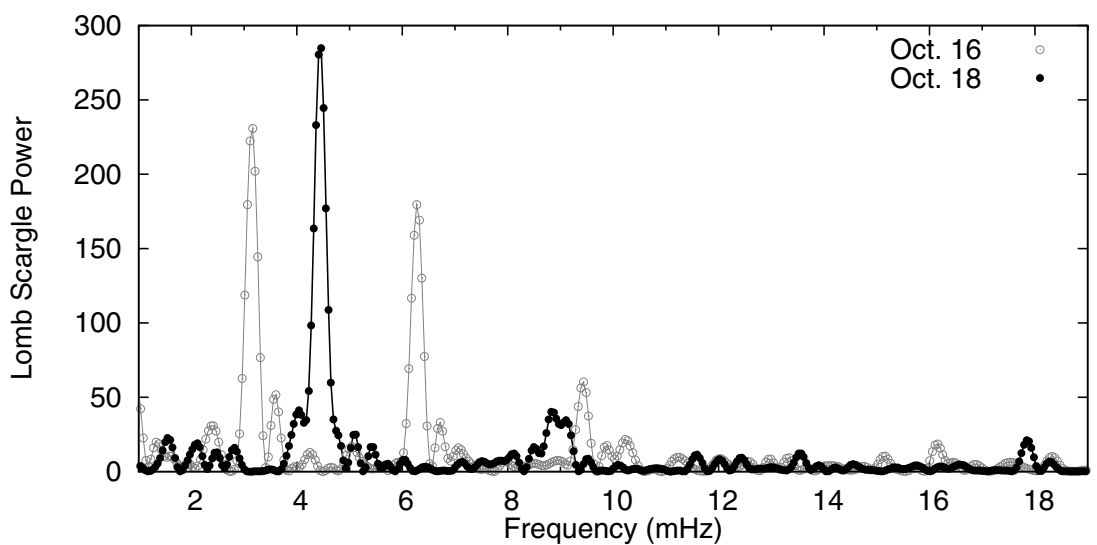

Figure 4. Lomb-Scargle periodograms of the $\mathrm{mHz}$ QPOs on October 16 (gray) and 18 (black) showing the QPO harmonic structure and the change in $v_{\mathrm{QPO}}$.

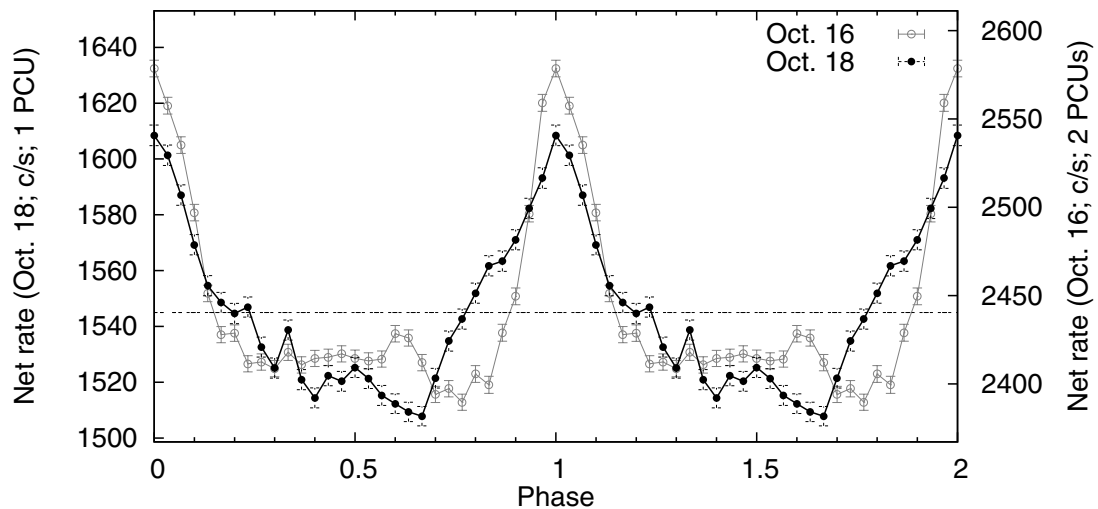

Figure 5. Folded background-subtracted 2-60 keV light curves of the $\mathrm{mHz}$ QPOs on October 16 (gray) and 18 (black). Peaks are at phase 0 by definition. Two QPO cycles are shown for clarity. Two PCUs were active on October 16, yielding a higher collected rate. The dashed horizontal line shows the average count rate, and the vertical range corresponds to $10 \%$ of that value in each case, showing that the fractional amplitude was higher on October 16 (Table 2). The net peak burst luminosity was in both cases $\simeq 1.3 \times 10^{37} \mathrm{erg} \mathrm{s}^{-1}$ (Table 1$)$.

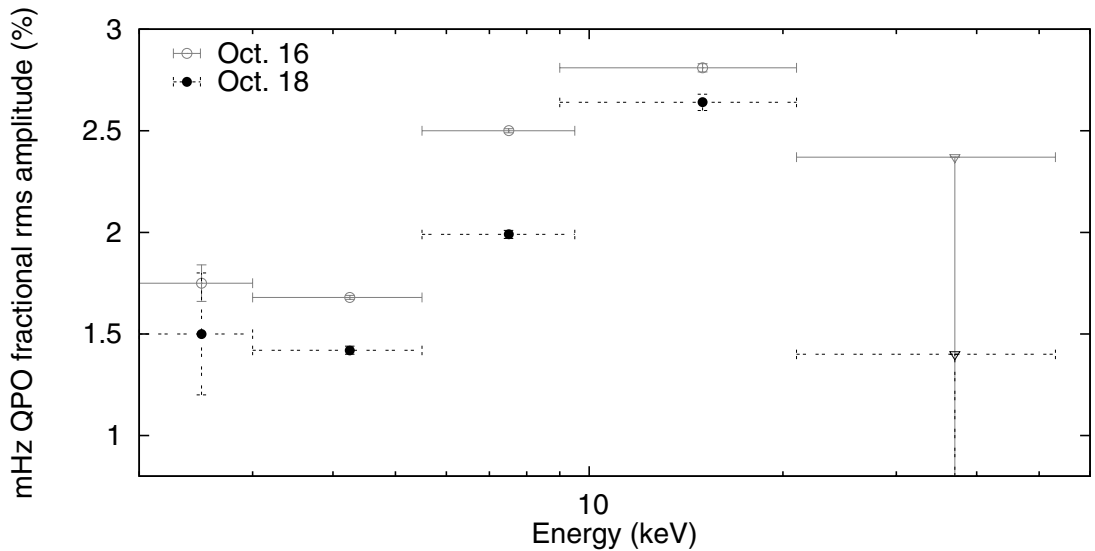

Figure 6. "rms spectrum" of the $\mathrm{mHz}$ QPOs on October 16 (gray) and 18 (black). Their fractional rms amplitude increases with energy up to $\sim 20 \mathrm{keV}$. No mHz QPOs are detected above that energy, empty triangles show upper limits on their fractional rms amplitude.

(Fujimoto et al. 1981; Bildsten 1998) much more closely than any other known burster (Cornelisse et al. 2003; Galloway et al. 2008). We expand on this comparison between thermonuclear burning theory and T5X2 in Section 4.1.

Moreover, T5X2 is the first slow X-ray pulsar (spin period $P_{\mathrm{s}}>10 \mathrm{~ms}$ ) to show thermonuclear bursts. None of the slower $\left(P_{\mathrm{s}} \gtrsim 1 \mathrm{~s}\right)$ "classical" X-ray pulsars in HMXBs have shown thermonuclear bursts to date, even if their persistent luminosity varies over the same range as in bursters. This is usually attributed to the stronger dipolar NS magnetic field $(B)$ in
HMXBs channeling the accretion flow into a much smaller area than LMXBs, leading to stable burning of all the accreted fuel due to a very high local $\dot{m}$ (Joss \& Li 1980; see also Section 4.3). On the other hand, only 7 out of the more than 90 bursters known have shown X-ray pulsations in the persistent emission (i.e., 7 out of the 14 accreting millisecond pulsars have shown bursts to date). Before the discovery of T5X2 the slowest spinning burster had a spin frequency more than 20 times higher than T5X2 (IGR J17511-3057, with a spin frequency $v_{\mathrm{s}}=245 \mathrm{~Hz}$; Markwardt et al. 2009; Altamirano et al. 2010c). T5X2 therefore 
Table 4

Summary of Bursting Regimes in T5X2

\begin{tabular}{lccccl}
\hline \hline Regime & $\begin{array}{c}L_{\text {pers }} \\
\left(L_{\text {Edd }}\right)\end{array}$ & $\begin{array}{c}t_{\text {rec }} \\
(\mathrm{s})\end{array}$ & $\begin{array}{c}E_{\mathrm{b}} \\
\left(10^{39} \mathrm{erg}\right)\end{array}$ & $\begin{array}{c}L_{\text {peak }} \\
\left(10^{37} \mathrm{erg} \mathrm{s}^{-1}\right)\end{array}$ & \multicolumn{1}{c}{ Description } \\
\hline A & $0.1-0.2$ & $>2000$ & $1.6-1.3$ & $4.6-3.7$ & $\begin{array}{l}\text { Slow decrease of } E_{\mathrm{b}} \text { and } L_{\text {peak }} \\
\left.\text { (and } t_{\text {rec }} \text { ? }\right) \text { with increasing } L_{\text {pers }}\end{array}$ \\
B & $0.2-0.3$ & $2000-400$ & $1.3-0.3$ & $3.7-1.2$ & $\begin{array}{l}\text { Fast drop of } t_{\text {rec }}, E_{\mathrm{b}}, \text { and } L_{\text {peak }} \\
\text { with increasing } L_{\text {pers. }} t_{\text {rec }} \propto L_{\text {pers }}^{-3}\end{array}$ \\
C & $0.3-0.5$ & $400-200$ & $\sim 0.3$ & $\sim 1.2$ & $\begin{array}{l}\text { Bursts evolve into mHz QPOs with } \\
\text { increasing frequency }\left(t_{\text {rec }} \propto L_{\text {pers }}^{-1}\right) .\end{array}$ \\
D & $0.3-0.5$ & $\ldots$ & $\ldots$ & $\ldots$ & $\begin{array}{l}E_{\mathrm{b}} \text { and } L_{\text {peak }} \text { "saturate" } \\
\text { No bursts } / \text { mHz QPOs detected on } \\
\text { normal and flaring branch in } Z \text { source phase }\end{array}$
\end{tabular}

Note. See Section 3.2 for details.

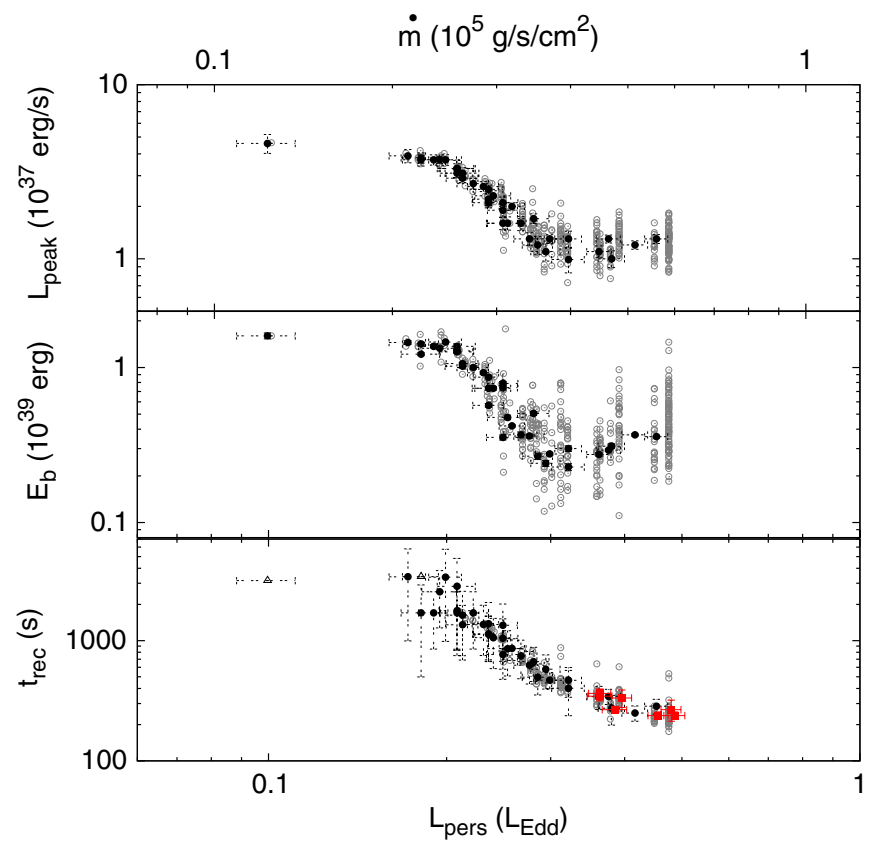

Figure 7. Burst peak luminosity (top), integrated burst energy (middle), and burst recurrence time, $t_{\text {rec }}$ (bottom) vs. bolometric persistent luminosity ( $L_{\text {pers }}$ ). Gray and black circles show individual burst measurements and daily averages, respectively. Red filled squares show recurrence times measured from the inverse of the $\mathrm{mHz}$ QPO frequency. The top axis shows the inferred $\dot{m}$ assuming homogeneous accretion onto a $1.4 M_{\odot}, 10 \mathrm{~km}$ radius NS (Section 2).

(A color version of this figure is available in the online journal.)

bridges the gap between "pulsars that do not burst and bursters that do not (typically) pulse" (Bildsten 1998 and references therein). We show this in Figure 10 by comparing both the $B$ and $P_{\mathrm{S}}$ values of T5X2 to those of LMXBs (including a few peculiar LMXBs) and HMXBs. Figure 10 clearly shows that T5X2 features values of $B$ and $P_{\mathrm{s}}$ intermediate between bursters and HMXBs. We discuss the consequences of such a high $B$ and long $P_{\mathrm{s}}$ for thermonuclear burst regimes in Sections 4.3 and 4.4, respectively.

The shortest burst wait times known to date (Linares et al. 2009; Keek et al. 2010; 5.4 and 3.8 minutes, respectively) were based on sets of typically two or three consecutive bursts followed by much longer periods without bursts. The bursts from $\mathrm{T} 5 \mathrm{X} 2$ presented herein are remarkably quasi-periodic, not only in the $\mathrm{mHz}$ QPO phase, as witnessed by the well-defined and smoothly evolving wait time in individual bursts $\left(t_{\text {wait }}\right.$; see Figures 1 and 2). This behavior is analogous to the "clocked

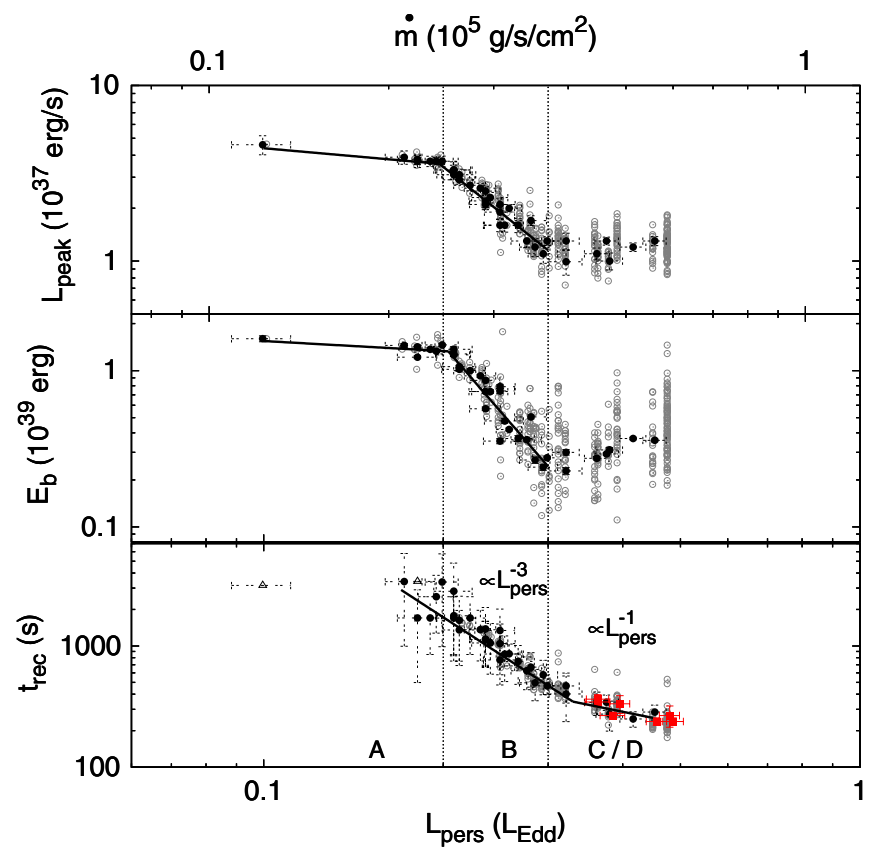

Figure 8. Same as Figure 7, showing also the broken power-law fits to the $L_{\text {peak }}, E_{\mathrm{b}}, t_{\text {rec }}$ vs. $L_{\text {pers }}$ relations (Table 3 ). Three regimes are apparent and labeled along the bottom axis: slow decrease (at $L_{\text {pers }} / L_{\text {Edd }} \lesssim 0.2$; regime A), fast drop (at $0.2 \lesssim L_{\text {pers }} / L_{\text {Edd }} \lesssim 0.3$; regime B), and saturation $\left(L_{\text {pers }} / L_{\text {Edd }} \gtrsim 0.3\right.$; regime C) of the $L_{\text {peak }}, E_{\mathrm{b}}$ vs. $L_{\text {pers }}$ relations. The $t_{\text {rec }}$ values follow approximate $t_{\text {rec }} \propto L_{\text {pers }}^{-3}$ and $t_{\text {rec }} \propto L_{\text {pers }}^{-1}$ relations in regimes B and C, as indicated (see Figure 11, Table 4, and Sections 3.2 and 4).

(A color version of this figure is available in the online journal.)

burster" (GS 1826-24; Tanaka 1989; Ubertini et al. 1999; Galloway et al. 2004; Heger et al. 2007a) and IGR J17511-3057 (Falanga et al. 2011), which have shown bursts at regular intervals with an approximate $t_{\text {rec }} \propto L_{\text {pers }}^{-1}$ relation, although at lower accretion rates than T5X2. For this reason, we compare in Figure 11 the burst energies and recurrence times of T5X2 to those of these two sources, as well as Cir X-1. The bursts from T5X2 (at $\dot{m} \sim 0.1-0.5 \dot{m}_{\mathrm{Edd}}$ ) are more frequent and less energetic than those from GS 1826-24 and IGR J17511-3057 (at $\dot{m} \sim 0.02-0.06 \dot{m}_{\text {Edd }}$ ). Figure 11 also shows that the $t_{\text {rec }} \propto$ $L_{\text {pers }}^{-3}$ relation that we find in regime B is unique to $55 \mathrm{X} 2$. The bursts from $\mathrm{Cir} X-1$, however, again have a strong resemblance to those of T5X2, with energies in the same range $\left(\lesssim 10^{39} \mathrm{erg}\right)$ and a similar $E_{\mathrm{b}}-L_{\text {pers }}$ relation (a break or transition is also suggested by the Cir X-1 data; Figure 11). 


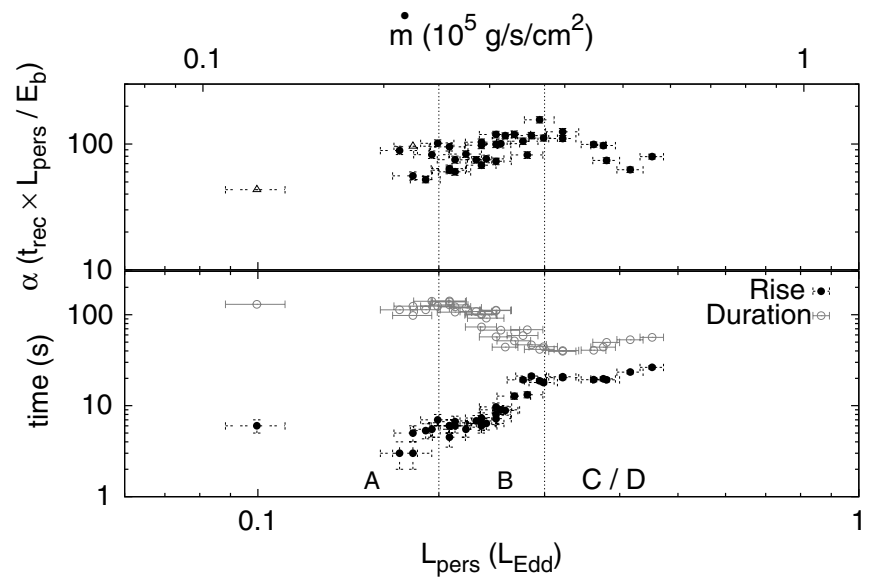

Figure 9. Daily-averaged accretion-to-burst energy ratio ( $\alpha$, top panel) and burst timescales (rise time and duration, bottom panel) vs. persistent luminosity ( $L_{\text {pers }}$ ) during the T5X2 outburst. Open triangles represent lower limits (one single burst detected during that day). The corresponding bursting regimes are indicated with dotted lines and labels along the bottom axis (Table 4).

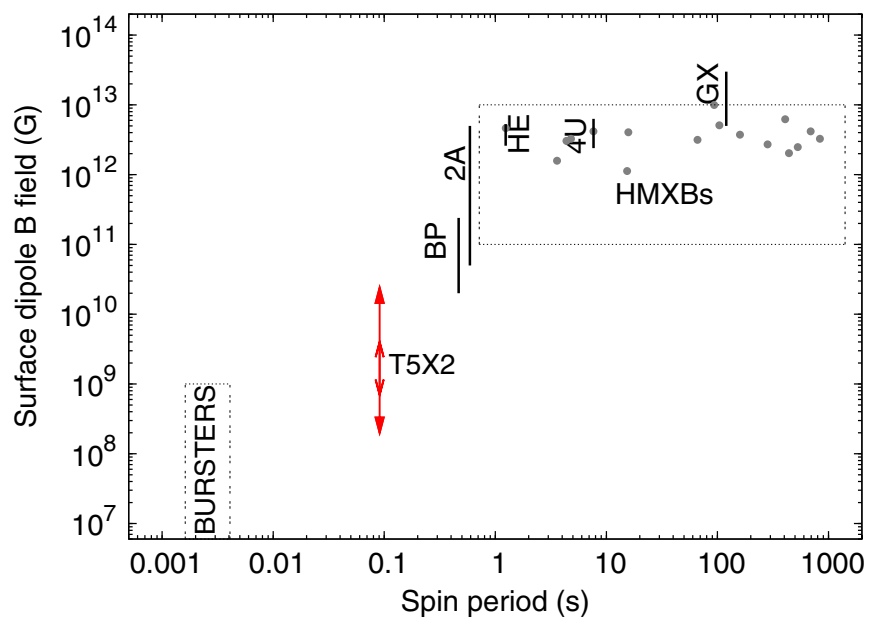

Figure 10. Magnetic field strength vs. spin period in accreting NSs. The dotted rectangle in the lower left corner (labeled "bursters") shows the location of all previously know thermonuclear burst sources with measured spin (Psaltis \& Chakrabarty 1999; Chakrabarty et al. 2003; Patruno 2010 and references therein). The dotted rectangle in the upper right corner (labeled "HMXBs") shows the location of X-ray pulsars in high-mass X-ray binaries (Bildsten et al. 1997; filled gray circles show X-ray pulsars with $B$ measured from cyclotron lines; Caballero \& Wilms 2011). Red double-headed arrows show the location of T5X2 (using the two magnetic field estimates from Papitto et al. 2011; Miller et al. 2011). Vertical black lines mark the location of other slow pulsars in LMXBs $\left(P_{\mathrm{s}}>0.01 \mathrm{~s}\right)$, none of which has shown thermonuclear bursts to date. These are labeled with the first two characters of the source names, which follow: 2A 1822-371 (Jonker \& van der Klis 2001), 4U 1626-67 (Rappaport et al. 1977; Orlandini et al. 1998), GRO 1744-28 (the "bursting pulsar" (BP); Kouveliotou et al. 1996; Finger et al. 1996; Cui 1997; Sturner \& Dermer 1996), Her X-1 (Truemper et al. 1978; Bildsten et al. 1997; Mihara et al. 1990; dal Fiume et al. 1998), and GX 1+4 (Chakrabarty \& Roche 1997; Hinkle et al. 2006; Cui 1997).

(A color version of this figure is available in the online journal.)

\subsection{Bursting Regimes versus Burning Regimes: The Need for Heat}

In this section we compare in detail the $\mathrm{T} 5 \mathrm{X} 2$ burst properties with theoretical predictions. Models of nuclear burning in the envelope of (non-magnetic, non-rotating) accreting NSs predict four different burning regimes on an NS accreting a mixture of hydrogen $\left(\mathrm{H}\right.$; mass fraction $\left.X_{0}\right)$, helium (He; mass fraction $Y_{0}$ ), and heavy elements (mainly CNO, mass fraction Z; see Woosley \& Taam 1976; Fujimoto et al. 1981;

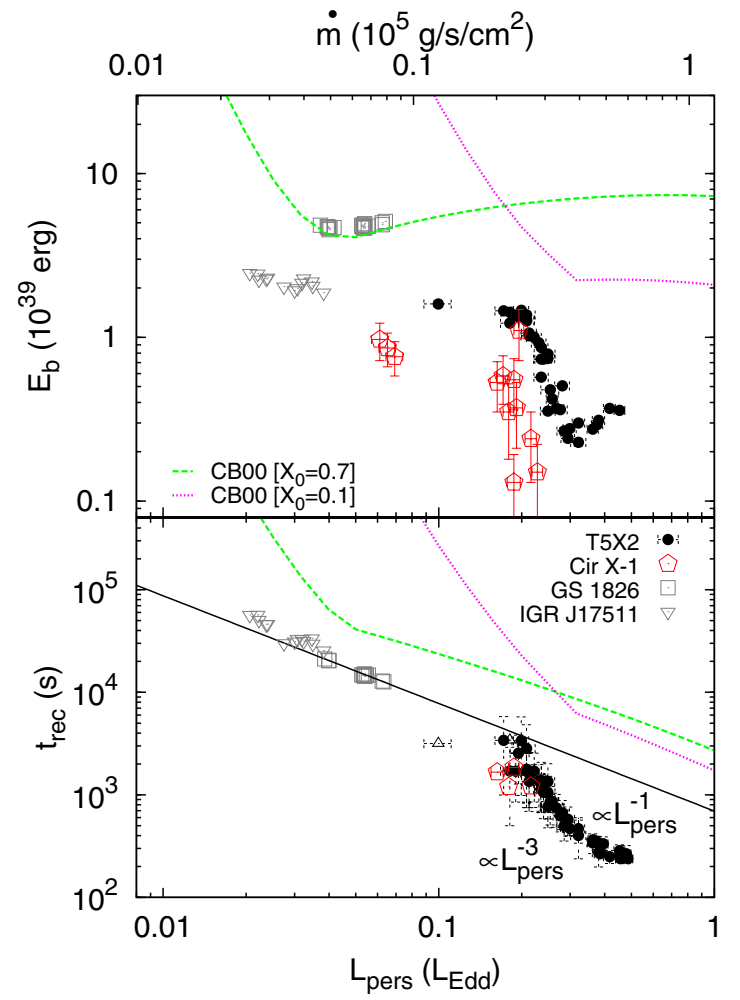

Figure 11. Burst energy (top panel) and recurrence time (bottom panel) vs. persistent luminosity (bottom axis) and inferred mass accretion rate per unit surface (top axis) for four bursters, as indicated on the bottom panel: T5X2 (filled black circles; this work), GS 1826-24 ("the clocked burster," open gray squares; from Galloway et al. 2004), Cir X-1 (open red pentagons; from Linares et al. 2010b), and IGR J17511-3057 (open gray triangles; from Falanga et al. 2011). The solid line shows the empirical $t_{\text {rec }} \propto L_{\text {pers }}^{-1.05}$ relation found for GS 1826- 24 by Galloway et al. (2004), similar to the $t_{\text {rec }} \propto L_{\text {pers }}^{-1.1}$ relation found in IGR J17511-3057 (Falanga et al. 2011). We also show the $E_{\mathrm{b}}-\dot{m}$ and $t_{\mathrm{rec}}-\dot{m}$ relations predicted by two ignition models from Cumming \& Bildsten (2000, labeled CB00) with different accreted hydrogen fractions: $X_{0}=0.7$ (green dashed line) and $X_{0}=0.1$ (magenta dotted line). In both models the metallicity was assumed to be $Z=0.02$ and the heat flux from the crust was fixed at $Q_{\mathrm{b}}=$ $0.1 \mathrm{MeV}$ nucleon $^{-1}$.

(A color version of this figure is available in the online journal.)

Taam 1981; Bildsten 1998; Cumming \& Bildsten 2000, and references therein). At the highest accretion rates, close to or higher than $\dot{m}_{\text {Edd }}$, both $\mathrm{H}$ and He burn stably and no bursts are expected (Section 4.2). For accretion rates

$$
\dot{m}>\dot{m}_{\mathrm{sHb}} \simeq 0.008 \dot{m}_{\mathrm{Edd}}\left(\frac{0.7}{X_{0}}\right)\left(\frac{Z}{0.01}\right)^{1 / 2},
$$

(Bildsten 1998; where we assumed an opacity of $0.04 \mathrm{~cm}^{2} \mathrm{~g}^{-1}$ and the value of $\dot{m}_{\text {Edd }}$ given in Section 2), H burns stably between bursts at a constant rate, via the so-called ( $\beta$-limited) hot-CNO cycle (Bildsten 1998; Cumming \& Bildsten 2000, and references therein). In this range all bursts are triggered when He burning at the base of the accreted layer becomes thermally unstable (He ignition). The column depth (or density) at the base of the burning layer when ignition occurs is known as ignition depth, $y_{\text {ign }}$, and the time between bursts is simply $t_{\text {rec }}=y_{\text {ign }} / \dot{m}$.

As $\mathrm{H}$ burns at a constant rate (temperature and $\dot{m}$ independent, but proportional to $Z$ ), the time it takes to consume all $\mathrm{H}$ in a sinking fluid element depends only on $X_{0}$ and $Z$ :

$$
t_{\text {burn }}=27 \mathrm{hr}\left(\frac{X_{0}}{0.7}\right)\left(\frac{0.01}{Z}\right)(1+z) / 1.31
$$


(as seen by an observer; e.g., Galloway \& Cumming 2006), where $1+z=\left(1-2 G M / R c^{2}\right)^{-1 / 2}=1.31$ is the gravitational redshift on the surface of a NS with mass $M=1.4 M_{\odot}$ and radius $R=10 \mathrm{~km}$. The longest burst recurrence time that we measure in T5X2 is $t_{\text {rec }} \sim 1 \mathrm{hr}$. For solar abundances this implies that $t_{\text {rec }} \ll t_{\text {burn }}$, i.e., there is no time to consume all $\mathrm{H}$ between the T5X2 bursts unless the fuel is substantially H-poor and/or metal-rich (see below). In general, for accretion rates

$$
\dot{m}>\dot{m}_{\mathrm{dep}} \simeq 0.04 \dot{m}_{\mathrm{Edd}}\left(\frac{0.7}{X_{0}}\right)\left(\frac{Z}{0.01}\right)^{13 / 18}
$$

(Bildsten 1998; for $M=1.4 M_{\odot}$ and $R=10 \mathrm{~km}$ ), there is no time to burn the accreted $\mathrm{H}$ before reaching ignition conditions (i.e., $t_{\text {rec }}<t_{\text {burn }}$ ) so that He ignites in a mixture of $\mathrm{H}$ and He. In this regime $y_{\text {ign }}$ does not depend sensitively on $\dot{m}$, and a simple $t_{\text {rec }} \propto \dot{m}^{-1}$ relation is expected. For $\dot{m}<\dot{m}_{\text {dep }}$ instead, there is enough time to deplete all $\mathrm{H}$ before the base of the accreted layer reaches ignition conditions. Bursts are then triggered in the absence of $\mathrm{H}$ (pure He ignition). In this regime $y_{\text {ign }}$ decreases with increasing $\dot{m}$, which results in a steeper decline of the burst recurrence time as $\dot{m}$ increases, close to $t_{\text {rec }} \propto \dot{m}^{-3}$ (Cumming $\&$ Bildsten 2000).

This transition between pure $\mathrm{He}$ and mixed $\mathrm{H} / \mathrm{He}$ ignition regimes can be seen in the $E_{\mathrm{b}}-\dot{m}$ and $t_{\mathrm{rec}}-\dot{m}$ relations in Figure 11, where we show two semi-analytic models from Cumming \& Bildsten (2000) with different compositions. Even though the transition from pure $\mathrm{He}$ to mixed $\mathrm{H} / \mathrm{He}$ ignition is expected at $\dot{m}_{\text {dep }} \sim 0.05 \dot{m}_{\text {Edd }}$ for the case of solar abundances, Figure 11 and Equation (3) clearly show that changes in the accreted composition can increase $\dot{m}_{\text {dep }}$ by a factor of at least 10. In particular, Figure 11 shows that ignition models with low-H abundance, $\left[X_{0}=0.1, Z=0.02\right]$, can reproduce the change in slope of both the $E_{\mathrm{b}}-L_{\text {pers }}$ and $t_{\mathrm{rec}}-L_{\text {pers }}$ relations that we observe in T5X2 at $L_{\text {pers }} \simeq 0.3 L_{\text {Edd }}$, i.e., at the transition between regimes B and C (Section 3.2, Tables 3 and 4). With such low $X_{0}, \mathrm{H}$ can be depleted before reaching He ignition at accretion rates much higher than in the case of solar abundances, which highlights the importance of fuel composition in the burning regimes.

The average $\mathrm{T} 5 \mathrm{X} 2$ accretion-to-burst energy ratio $(\alpha=$ $\left.Q_{\text {grav }}(1+z) / Q_{\text {nuc }}\right),\langle\alpha\rangle=102$, corresponds to a total nuclear energy release during the bursts $Q_{\text {nuc }} \simeq 2.8 \mathrm{MeV}_{\text {nucleon }^{-1}}$ (see also Motta et al. 2011). Taking $Q_{\text {nuc }}=1.6+4\langle X\rangle \mathrm{MeV}$ nucleon $^{-1}$ (which assumes complete burning of the accumulated fuel and $\sim 35 \%$ neutrino energy loss; Galloway et al. 2008 and references therein) we find an average $\mathrm{H}$ mass fraction over the burning layer $\langle X\rangle \simeq 0.3$. Such low inferred $Q_{\text {nuc }}$ lends support to the low-H fraction fuel scenario for T5X2 proposed above. In summary, the slopes of the $E_{\mathrm{b}}-L_{\text {pers }}$ and $t_{\text {rec }}-L_{\text {pers }}$ relations as well as the $\alpha$ values observed during the T5X2 outburst indicate a low accreted $\mathrm{H}$ fraction and strongly suggest a transition from the pure He ignition regime to the mixed $\mathrm{H} / \mathrm{He}$ ignition regime happening during the outburst rise when $\dot{m}$ increases above $\sim 0.3 \dot{m}_{\text {Edd }}$. It is worth noting that the reverse transition is observed during the outburst decay when $\dot{m}$ drops below $0.3 \dot{m}_{\text {Edd }}$ (i.e., the outburst rise and decay tracks overlap and no hysteresis is seen in Figures 7 and 8).

We stress that the link between the observed T5X2 bursting regimes and the theoretical burning regimes that we put forward is based on the $t_{\text {rec }}-L_{\text {pers }}$ and $E_{\mathrm{b}}-L_{\text {pers }}$ relations. Modeling and interpretation of the individual burst light curves is beyond the scope of this work, yet we note that different burst light curves and peak luminosities are predicted in the different ignition regimes (e.g., Woosley et al. 2004). Pure He bursts are expected to show faster rise times than mixed $\mathrm{H} / \mathrm{He}$ bursts. This agrees qualitatively with the identification of regime $\mathrm{B}$ as pure $\mathrm{He}$ ignition: $t_{\text {rise }}$ is shorter in regime $\mathrm{B}$ than in regime $\mathrm{C}$ (Figure 9). The large change in $L_{\text {pers }}$, however, could influence the observed timescales along the burst rise and decay, as these are measured after subtracting the persistent emission. It should also be noted that at low accretion rates (near $\dot{m}_{\mathrm{sHb}} \simeq 0.08 \dot{m}_{\mathrm{Edd}}$ for $X_{0}=0.1$, $Z=0.02$ ) there should be a transition to H-ignited bursts, not included in the models discussed in this section (Cumming \& Bildsten 2000).

If the link between bursting and burning regimes that we propose is correct, to our knowledge this is the first time that the transition between pure $\mathrm{He}$ and mixed $\mathrm{H} / \mathrm{He}$ ignition is observed in a single source. There is, however, a systematic and interesting discrepancy evident in Figure 11. Even when including compressional heating or when increasing the base heat flux from the NS crust (up to $2 \mathrm{MeV}^{\text {nucleon }}{ }^{-1}$; fixed at $0.1 \mathrm{MeV}^{\text {nucleon }}{ }^{-1}$ in Figure 11; Cumming \& Bildsten 2000), ignition models predict higher burst energies and longer recurrence times than those we find in $\mathrm{T} 5 \mathrm{X} 2$, by a factor close to 10 in most cases (i.e., larger than the distance uncertainty on $L_{\text {pers }}$ and $E_{\mathrm{b}}$, Section 2). This large difference between the observed and predicted values of $E_{\mathrm{b}}$ and $t_{\text {rec }}$, together with the lack of detailed modeling of the T5X2 burst light curves, prevents a conclusive identification of the observed bursting regimes.

We propose that such discrepancy could be explained by the presence of an extra source of heat in the NS envelope not accounted for by ignition models (which typically consider only hot-CNO heating). Additional heat would act to reduce $y_{\text {ign }}$ and thereby decrease $E_{\mathrm{b}}$ and $t_{\mathrm{rec}}$, explaining the T5X2 observations presented herein. Several interesting possibilities for the nature of this extra source of heat have been partially investigated, including: (1) heating due to stable He burning (triple alpha reaction rates have been recently debated; e.g., Ogata et al. 2009; Dotter \& Paxton 2009; Peng \& Ott 2010); (2) heating due to deep burning of residual H (e.g., Taam et al. 1996, who already pointed out that it can lead to weaker and more frequent bursts than expected); (3) thermal inertia, "hot ashes" or heat from previous bursts, which could become important for T5X2 as it features $t_{\mathrm{rec}} \sim 200 \mathrm{~s}$, the shortest recurrence times between thermonuclear bursts ever observed (time-dependent simulations of a series of bursts are needed to investigate this in detail; e.g., Heger et al. 2007b); and (4) turbulent friction at the base of the spreading layer, which could release substantial amounts of heat (Inogamov \& Sunyaev 2010). It is worth noting that an independent study of the same source (Degenaar et al. 2011) reached similar conclusions, suggesting the presence of a "shallow heat source" in T5X2 in order to reconcile quiescent observations and crust heating/cooling theory. Without regard to the exact nature of this heat source, we have shown in the present work that the burst properties of T5X 2 place new constraints on the thermal properties of a fast-accreting and frequently bursting NS.

\section{2. $\mathrm{mHz}$ QPOs and Marginally Stable Burning}

The $\mathrm{mHz}$ QPOs from T5X2 (Section 3.1; see also Linares et al. 2010a) have distinctive properties that clearly set them apart from the previously known $\mathrm{mHz}$ QPOs (Section 1; Revnivtsev et al. 2001; Altamirano et al. 2008). The persistent luminosity that we measure in $\mathrm{T} 5 \mathrm{X} 2 \mathrm{when} \mathrm{mHz}$ QPOs are 
present is about 10 times higher than that observed in previous $\mathrm{mHz}$ QPO sources ( $L_{\text {pers }}$ higher by a factor of $4-25$ taking into account the observed ranges: $0.02-0.1 L_{\text {Edd }}$ in atoll sources as opposed to $0.4-0.5 L_{\text {Edd }}$ in T5X2; Table 2). For this reason we refer to the previously known $\mathrm{mHz}$ QPOs as "low- $L_{\text {pers }}$ mHz QPOs."

Bright bursts and low- $L_{\text {pers }} \mathrm{mHz}$ QPOs alternate, while remaining clearly distinguishable. Instead, in T5X2 bursts smoothly evolve into $\mathrm{mHz}$ QPOs and vice versa (Section 3.1), a phenomenon never observed before. Strikingly, the same qualitative evolution from bright infrequent bursts to faint and frequent bursts, $\mathrm{mHz}$ QPOs, and ultimately stable burning is predicted to happen as $\dot{m}$ increases by both one-zone models and detailed simulations of nuclear burning on NSs accreting near the boundary between unstable and stable He burning (Heger et al. 2007b). We can therefore identify with confidence the $\mathrm{mHz}$ QPOs from T5X2 with marginally stable burning on the NS surface. The evidence that links low- $L_{\text {pers }} \mathrm{mHz}$ QPOs with marginally stable burning is less conclusive, but remains valid (see Revnivtsev et al. 2001; Yu \& van der Klis 2002; Altamirano et al. 2008 for details).

Analytic estimates place the threshold for stable He burning at

$$
\dot{m}_{\mathrm{sb}} \simeq 1.1 \dot{m}_{\mathrm{Edd}}\left(\frac{1.7}{1+X_{0}}\right)^{3 / 4}\left(\frac{Y_{0} \mu}{0.3 \times 0.6}\right)^{1 / 2}
$$

where $\mu$ is the mean molecular weight of the accreted fuel (Bildsten 1998; assuming again $M=1.4 M_{\odot}$ and $R=10 \mathrm{~km}$ ). The persistent luminosity where $\mathrm{mHz}$ QPOs are observed in T5X2 $\left(0.4-0.5 L_{\text {Edd }}\right)$ is therefore closer to the expected value of $\dot{m}_{\mathrm{sb}}$ than what was seen in low- $L_{\text {pers }} \mathrm{mHz}$ QPOs, yet still inconsistent with the value predicted by theory, which is higher by a factor of $\sim 2$ (unless Terzan 5 is at $\sim 9 \mathrm{kpc}$ instead of $6.3 \mathrm{kpc}$, Section 2). Invoking H-poor fuel makes this discrepancy even larger, as it can increase the expected $\dot{m}_{\mathrm{sb}}$ by a factor two (for $X_{0}=0.1, Z=0.01$, Equation (4)). A more massive NS can have unstable burning at higher $\dot{m}$ than a less massive star, but given the weak $\left(M^{1 / 2}\right)$ scaling of $\dot{m}_{\mathrm{sb}}$ different NS masses cannot explain the large difference (factor 4-25) in $L_{\text {pers }}$ between T5X2 and the other known sources of $\mathrm{mHz}$ QPOs. Another remarkable difference between the $\mathrm{mHz}$ QPOs presented herein and the low- $L_{\text {pers }} \mathrm{mHz}$ QPOs resides in their fractional rms spectrum (Section 2): we find a fractional rms amplitude that increases with energy between 2 and $20 \mathrm{keV}$ (Section 3.1), while Revnivtsev et al. (2001) reported fractional amplitudes decreasing with increasing photon energy between 2 and $5 \mathrm{keV}$. The energy-averaged fractional amplitudes are, however, similar $\left(1.3 \%-2.2 \%\right.$ in $\mathrm{T} 5 \mathrm{X} 2$; this work; $0.7 \%-1.9 \%$ in low- $L_{\text {pers }}$ $\mathrm{mHz}$ QPOs; Revnivtsev et al. 2001). The increase of fractional amplitude with energy that we find is shallower than the linear increase predicted by models of temperature oscillations at the NS surface (developed in the context of burst oscillations; Piro \& Bildsten 2006).

An interesting possibility is that the low- $L_{\text {pers }} \mathrm{mHz}$ QPOs trace the boundary of stable $\mathrm{H}$ burning (Equation (1)), whereas the $\mathrm{mHz}$ QPOs that we discovered in T5X2, at a higher accretion rate, occur at the boundary of stable He burning (Equation (4)). This remains speculative at present given the lack of published theoretical work on this particular topic. A specific analysis of oscillatory burning at the thermal stability boundary of $\mathrm{H}$ burning is needed to address this hypothesis. The oscillatory behavior at the marginally stable point is generic (Paczynski 1983 ) and it could also occur when H burning stabilizes. If low-
$L_{\text {pers }} \mathrm{mHz}$ QPOs do happen at the H-burning stability boundary, the need to invoke confined accretion to explain their low $L_{\text {pers }}$ would vanish.

Heger et al. (2007b) found that the QPO frequency is mainly sensitive to the accreted $\mathrm{H}$ fraction $\left(X_{0}\right)$ and the NS surface gravity: increasing the surface gravity or decreasing $X_{0}$ leads to higher $\mathrm{mHz} \mathrm{QPO}$ frequencies. The highest $v_{\mathrm{QPO}}$ values that we find in T5X2 $(4.2 \mathrm{mHz})$ are about a factor of three lower than those of the low- $L_{\text {pers }} \mathrm{mHz}$ QPOs (Table 2; Revnivtsev et al. 2001; Altamirano et al. 2008). If the accreted fuel has a similar composition, and if the $\mathrm{mHz}$ QPOs have the same origin, this would suggest a less compact NS in T5X2 than in the low- $L_{\text {pers }} \mathrm{mHz}$ QPO sources (the "atoll" sources 4U 1636-536, 4U 1608-52, and Aql X-1). Comparing with models of marginally stable burning, we find that if $X_{0}<0.7$ the surface gravity of the NS in T5X2 must be $g=G M / R^{2}<$ $1.9 \times 10^{14} \mathrm{~cm} \mathrm{~s}^{-2}$ (Figure 9 in Heger et al. 2007b). Even though it is subject to theoretical (based on analytic one-zone model) and observational (a new outburst of T5X2 could show higher $v_{\mathrm{QPO}}$ ) uncertainties, this illustrates how a comparison between marginally stable burning models and $\mathrm{mHz}$ QPO properties can be used to constrain the NS compactness.

Despite the striking similarities between the $\mathrm{T} 5 \mathrm{X} 2$ burst properties and the general bursting behavior predicted by models of nuclear burning near the transition from unstable to stable burning (cf. Figure 1 in this work and Figure 5 in Heger et al. 2007b), interesting differences remain. First, as explained above, the $\dot{m}$ where marginally stable burning is expected (0.925 $\dot{m}_{\text {Edd }}$; Heger et al. 2007b) is higher than the highest inferred $\dot{m}$ where we observe $\mathrm{mHz}$ QPOs in T5X2 $\left(0.5 \dot{m}_{\text {Edd }}\right)$. Second, Heger et al. (2007b) find a sharp transition from bursts to $\mathrm{mHz}$ QPOs and finally stable burning (occurring between $0.923-0.95 \dot{m}_{\text {Edd }}$ ), while we observe in T5X2 a smooth evolution from bursts to $\mathrm{mHz}$ QPOs, and vice versa (between 0.1 and $0.5 \dot{m}_{\text {Edd }}$; Section 3). Time-dependent simulations of nuclear burning tailored to T5X2 would be of high interest, in particular exploring the H-poor fuel range. Third, we find that the disappearance of the $\mathrm{mHz}$ QPOs in T5X2 is not linked to an increase in $\dot{m}$ but to an actual drop of $L_{\text {pers }}$ that happens when the accretion state changes (from horizontal to normal and flaring branches). This suggests that the geometry/configuration of the accretion flow plays a role in setting the nuclear burning stability boundary.

\subsection{The Role of an Intermediate Magnetic Field}

While most models of thermonuclear bursts on accreting NSs assume that the NS magnetic field is negligible (Fujimoto et al. 1981; Taam 1982; Paczynski 1983; Woosley et al. 2004), Joss $\& \mathrm{Li}(1980)$ showed that the presence of a strong $\left(B \gtrsim 10^{12} \mathrm{G}\right)$ magnetic field can affect the stability of nuclear burning in different ways. A strong magnetic field reduces the (conductive and radiative) opacities, allowing for more efficient heat transport and thereby stabilizing burning. Applying disk-magnetosphere interaction models to T5X2, Papitto et al. (2011) estimated $B=2 \times 10^{8}-2.4 \times 10^{10} \mathrm{G}$ from the luminosity range at which pulsations were detected, whereas Miller et al. (2011) further constrained $B=(0.7-4) \times 10^{9} \mathrm{G}$ from the Fe line profile. The fact that $\mathrm{T} 5 \mathrm{X} 2$ shows thermonuclear bursts suggests that the magnetic field needed to suppress the thermal instability and quench thermonuclear bursts must be greater than $\sim 10^{10} \mathrm{G}$, in accordance with theoretical expectations (Joss \& Li 1980). 
A strong magnetic field also affects convective heat transport and mixing (e.g., Joss \& Li 1980). Bildsten (1995) proposed that even in cases where thermonuclear burning is unstable the presence of a strong magnetic field can suppress convection in the NS envelope, stalling the propagation of the burning front (diffusive heat transport being slower than convection) and preventing the fast ignition that causes type I X-ray bursts (see also Bildsten 1998). Again, the mere presence of type I $\mathrm{X}$-ray bursts in T5X2 strongly suggests that the magnetic field required to suppress convective burning fronts must be higher than $\sim 10^{10} \mathrm{G}$, in agreement with analytical estimates (Joss \& Li 1980; Bildsten \& Brown 1997). Rise times are rather long however (Figure 9), which could perhaps indicate that $B$ is strong enough to slow down the burning fronts (Bildsten \& Brown 1997). In summary, the bursting properties of T5X2 presented herein (in particular combined with refined measurements of its magnetic field strength) can place new constraints on the physics of convection and heat transport under intermediate magnetic fields $\left(10^{8}-10^{10} \mathrm{G}\right)$.

Finally, a $10^{8}-10^{10} \mathrm{G}$ magnetic field can also channel the accretion flow onto the magnetic polar caps, as suggested by theoretical models of disk-magnetosphere interaction (Lamb et al. 1973) and by the presence of X-ray pulsations in T5X2 (Strohmayer \& Markwardt 2010; Papitto et al. 2011). Accretion confined to the magnetic polar caps may break the spherical symmetry assumed by most thermonuclear burst models, and increase $\dot{m}$ for a given (observed) $L_{\text {pers }}$ (Section 2). The impact on the burning properties depends on the size of the polar caps and on how soon the accreted fuel spreads laterally while sinking into the NS envelope under the influence of a magnetic field. Bildsten \& Brown (1997) found that for dipolar magnetic fields $B<(2-4) \times 10^{10} \mathrm{G}$, the accreted fuel spreads before igniting and the spherically symmetric case is recovered. This suggests that the T5X2 burst properties can still be compared to spherically symmetric ignition models (Section 4.1).

As the size and "depth" of the polar caps are nevertheless illconstrained quantities, we explore a simple scenario in which the fuel in T5X2 is confined into a $10 \%$ of the NS surface down to ignition depth, $y_{\text {ign }}$. From geometrical considerations, assuming the same $y_{\text {ign }}$ predicted by ignition models (Cumming \& Bildsten 2000), in this confined scenario bursts would be 10 times less energetic and 10 times more frequent than in the spherically symmetric case, which would explain the mismatch between predicted and observed $E_{\mathrm{b}}$ and $t_{\mathrm{rec}}$ (Section 4.1). However, the accretion rate per unit area would also increase by a factor 10 , which for the observed $L_{\text {pers }}$ implies that unstable burning would operate at $\dot{m}$ as high as $\sim 5$ times $\dot{m}_{\text {Edd }}$. This is well above the predicted threshold for stable steady burning of $\mathrm{H}$ and $\mathrm{He}\left(\dot{m}_{\mathrm{sb}}\right.$, Equation (4); see also Bildsten 1998), and we therefore consider this scenario unlikely. If the accreted material contains no $\mathrm{H}$, however, $\dot{m}_{\mathrm{sb}}$ can be several times larger than $\dot{m}_{\text {Edd }}$ (Bildsten 1998).

\subsection{Rotation, Mixing, and Burning}

The unique bursting behavior in T5X2 is, in essence, much closer to what theory predicts than any other burster known to date. Theoretical burning regimes were mostly based on models that assume a non-rotating NS and radial infall of the accreted mass (Fujimoto et al. 1981; Bildsten 1998). These are admittedly simplistic assumptions, as NSs in LMXBs can have spin frequencies in excess of $600 \mathrm{~Hz}$ and the orbital frequencies in the innermost accretion disk are well above $1000 \mathrm{~Hz}$. The accretion of matter with angular momentum onto a spinning
NS introduces a shear in its surface layers (Piro \& Bildsten 2007; Keek et al. 2009, and references therein). The main effect of rotation which may have an impact on burning regimes is turbulent mixing. In particular, Keek et al. (2009) found that such rotationally induced turbulent mixing stabilizes He burning, decreasing the burning stability boundary $\left(\dot{m}_{\mathrm{sb}}\right)$.

Motivated by the stronger magnetic field (factor $\sim 10$ ) and slower spin (factor $\gtrsim 20$ ) of T5X2 compared to the rest of bursters, we speculate that rotationally induced turbulent mixing is what sets T5X2 apart from the rest of thermonuclear burst sources. The stronger $B$ field could channel a large fraction of the accreted matter into the NS magnetic poles and produce a more radial inflow which would then spread over the whole surface, minimizing shear, and turbulent mixing. Therefore T5X2 seems to meet to a greater extent the assumptions of zero spin and radial infall mentioned above, which can explain the better agreement with theory. This in turn suggests that, as argued by Piro \& Bildsten (2007) and Keek et al. (2009), the effects of rotation must be considered to explain the behavior of most bursters.

\section{SUMMARY AND CONCLUSIONS}

We have presented the discovery of $\mathrm{mHz}$ QPOs from the NS-LMXB and $11 \mathrm{~Hz}$ X-ray pulsar T5X2, as well as the full bursting properties during its 2010 outburst, when the persistent luminosity varied by about a factor of five (between $\left.L_{\text {pers }} \simeq 0.1-0.5 L_{\text {Edd }}\right)$. T5X2 showed copious thermonuclear bursts when $L_{\text {pers }} \gtrsim 0.2 L_{\text {Edd }}$, a regime where thermonuclear bursts had proven exceptional to date. Burst energies and recurrence times gradually decreased as $L_{\text {pers }}$ increased, turning into a rapid series of faint bursts and smoothly evolving into a $\mathrm{mHz}$ QPO near the outburst peak. Only at the highest $L_{\text {pers }}$ range did bursts become undetectable during a few short intervals. This behavior is both unprecedented among bursters and remarkably similar to the nuclear burning regimes expected on an accreting NS near the boundary of stable He burning.

We find four different bursting regimes when studying the relation between burst properties and $L_{\text {pers }}$ and show that in one such regime the burst recurrence time decays steeply with increasing $L_{\text {pers }}$, close to $t_{\text {rec }} \propto L_{\text {pers }}^{-3}$. By confronting the change in burst properties (in particular, the relation between $E_{\mathrm{b}}, t_{\mathrm{rec}}$, and $L_{\text {pers }}$ ) to ignition model predictions, we find evidence of a transition between pure $\mathrm{He}$ and mixed $\mathrm{H} / \mathrm{He}$ ignition occurring in T5X2 when $L_{\text {pers }} \simeq 0.3 L_{\text {Edd }}$. We note that large discrepancies remain between the observed T5X2 burst properties and those predicted by theory at high-mass accretion rates. We further argue that the accreted fuel is H-poor and suggest that an additional source of heat in the NS envelope is needed to reconcile the observed and predicted burst properties.

We examine the properties of the $\mathrm{mHz}$ QPOs from T5X2 in the context of marginally stable burning models, and compare them to those of previously known $\mathrm{mHz}$ QPO sources. T5X2 features $\mathrm{mHz}$ QPOs with lower frequencies (by a factor $\sim 3$ ), when $L_{\text {pers }}$ is substantially higher (by a factor $\sim 4-25$ ). Finally, we discuss the role of magnetic field and spin in setting the unique T5X2 burst and $\mathrm{mHz}$ QPO behavior, and speculate that the absence of rotation effects such as turbulent mixing of the accreted fuel may set T5X2 apart from the rest of bursters.

We thank M. van der Klis for detailed and critical comments on the manuscript. We are grateful to the International Space Science Institute in Bern, where part of this work was completed. M.L. acknowledges support from the NWO Rubicon fellowship. 


\section{REFERENCES}

Altamirano, D., Homan, J., Linares, M., et al. 2010a, ATel, 2952, 1

Altamirano, D., van der Klis, M., Wijnands, R., \& Cumming, A. 2008, ApJ, 673, L35

Altamirano, D., Watts, A., Kalamkar, M., et al. 2010b, ATel, 2932, 1

Altamirano, D., Watts, A., Linares, M., et al. 2010c, MNRAS, 409, 1136

Belian, R. D., Conner, J. P., \& Evans, W. D. 1976, ApJ, 206, L135

Bildsten, L. 1995, ApJ, 438, 852

Bildsten, L. 1998, in NATO ASIC Proc. 515, The Many Faces of Neutron Stars., ed. R. Buccheri, J. van Paradijs, \& A. Alpar (Dordrecht: Kluwer), 419

Bildsten, L. 2000, in AIP Conf. Proc. 522, Cosmic Explosions, ed. S. S. Holt \& W. W. Zhang (Melville, NY: AIP), 359

Bildsten, L., \& Brown, E. F. 1997, ApJ, 477, 897

Bildsten, L., Chakrabarty, D., Chiu, J., et al. 1997, ApJS, 113, 367

Bordas, P., Kuulkers, E., Alfonso-Garzon, J., et al. 2010, ATel, 2919, 1

Caballero, I., \& Wilms, J. 2011, Mem. Soc. Astron. Ital., submitted

Cavecchi, Y., Patruno, A., Haskell, B., et al. 2011, ApJ, 740, L8

Chakrabarty, D., Morgan, E. H., Muno, M. P., et al. 2003, Nature, 424, 42

Chakrabarty, D., \& Roche, P. 1997, ApJ, 489, 254

Chakraborty, M., \& Bhattacharyya, S. 2011, ApJ, 730, L23

Chenevez, J., Kuulkers, E., Alfonso-Garzon, J., et al. 2010, ATel, 2924, 1

Cohn, H. N., Lugger, P. M., Grindlay, J. E., \& Edmonds, P. D. 2002, ApJ, 571, 818

Cornelisse, R., in't Zand, J. J. M., Verbunt, F., et al. 2003, A\&A, 405, 1033

Cui, W. 1997, ApJ, 482, L163

Cumming, A., \& Bildsten, L. 2000, ApJ, 544, 453

dal Fiume, D., Orlandini, M., Cusumano, G., et al. 1998, A\&A, 329, L41

Degenaar, N., Brown, E. F., \& Wijnands, R. 2011, MNRAS, 418, L152

Dotter, A., \& Paxton, B. 2009, A\&A, 507, 1617

Falanga, M., Kuiper, L., Poutanen, J., et al. 2011, A\&A, 529, A68

Finger, M. H., Koh, D. T., Nelson, R. W., et al. 1996, Nature, 381, 291

Fujimoto, M. Y., Hanawa, T., \& Miyaji, S. 1981, ApJ, 247, 267

Galloway, D. K., \& Cumming, A. 2006, ApJ, 652, 559

Galloway, D. K., Cumming, A., Kuulkers, E., et al. 2004, ApJ, 601, 466

Galloway, D. K., \& in't Zand, J. J. M. 2010, ATel, 3000, 1

Galloway, D. K., Muno, M. P., Hartman, J. M., Psaltis, D., \& Chakrabarty, D. 2008, ApJS, 179, 360

Grindlay, J., Gursky, H., Schnopper, H., et al. 1976, ApJ, 205, L127

Hasinger, G., \& van der Klis, M. 1989, A\&A, 225, 79

Heger, A., Cumming, A., Galloway, D. K., \& Woosley, S. E. 2007a, ApJ, 671, L141

Heger, A., Cumming, A., \& Woosley, S. E. 2007b, ApJ, 665, 1311

Heinke, C. O., Wijnands, R., Cohn, H. N., et al. 2006, ApJ, 651, 1098

Hinkle, K. H., Fekel, F. C., Joyce, R. R., et al. 2006, ApJ, 641, 479

Hoffman, J. A., Marshall, H. L., \& Lewin, W. H. G. 1978, Nature, 271, 630

Inogamov, N. A., \& Sunyaev, R. A. 2010, Astron. Lett., 36, 848

Jonker, P. G., \& van der Klis, M. 2001, ApJ, 553, L43

Joss, P. C., \& Li, F. K. 1980, ApJ, 238, 287

Keek, L., Galloway, D. K., in't Zand, J. J. M., \& Heger, A. 2010, ApJ, 718, 292

Keek, L., \& in't Zand, J. J. M. 2008, in Proc. 7th INTEGRAL Workshop, PoS(Integral08)032
Keek, L., Langer, N., \& in't Zand, J. J. M. 2009, A\&A, 502, 871

Kouveliotou, C., van Paradijs, J., Fishman, G. J., et al. 1996, Nature, 379, 799

Lamb, F. K., Pethick, C. J., \& Pines, D. 1973, ApJ, 184, 271

Lanzoni, B., Ferraro, F. R., Dalessandro, E., et al. 2010, ApJ, 717, 653

Lewin, W. H. G., \& Joss, P. C. 1977, Nature, 270, 211

Lewin, W. H. G., van Paradijs, J., \& Taam, R. E. 1993, Space Sci. Rev., 62, 223

Linares, M., Altamirano, D., Watts, A., et al. 2010a, ATel, 2958, 1

Linares, M., Chakrabarty, D., \& van der Klis, M. 2011, ApJ, 733, L17

Linares, M., Watts, A., Altamirano, D., et al. 2009, ATel, 1979, 1

Linares, M., Watts, A., Altamirano, D., et al. 2010b, ApJ, 719, L84

Lomb, N. R. 1976, Ap\&SS, 39, 447

Maraschi, L., \& Cavaliere, A. 1977, in X-Ray Binaries and Compact Objects, ed. K. A. van der Hucht, 127

Markwardt, C. B., Altamirano, D., Swank, J. H., et al. 2009, ATel, 2197, 1

Mihara, T., Makishima, K., Ohashi, T., Sakao, T., \& Tashiro, M. 1990, Nature, 346,250

Miller, J. M., Maitra, D., Cackett, E. M., Bhattacharyya, S., \& Strohmayer, T. E. 2011, ApJ, 731, L7

Motta, S., D’Aì, A., Papitto, A., et al. 2011, MNRAS, 414, 1508

Ogata, K., Kan, M., \& Kamimura, M. 2009, Prog. Theor. Phys., 122, 1055

Orlandini, M., Fiume, D. D., Frontera, F., et al. 1998, ApJ, 500, L163

Ortolani, S., Barbuy, B., Bica, E., Zoccali, M., \& Renzini, A. 2007, A\&A, 470, 1043

Paczynski, B. 1983, ApJ, 264, 282

Papitto, A., D'Aì, A., Motta, S., et al. 2011, A\&A, 526, L3

Patruno, A. 2010, ApJ, 722, 909

Peng, F., \& Ott, C. D. 2010, ApJ, 725, 309

Piro, A. L., \& Bildsten, L. 2006, ApJ, 638, 968

Piro, A. L., \& Bildsten, L. 2007, ApJ, 663, 1252

Pooley, D., Homan, J., Heinke, C., et al. 2010, ATel, 2974, 1

Psaltis, D., \& Chakrabarty, D. 1999, ApJ, 521, 332

Rappaport, S., Markert, T., Li, F. K., et al. 1977, ApJ, 217, L29

Revnivtsev, M., Churazov, E., Gilfanov, M., \& Sunyaev, R. 2001, A\&A, 372, 138

Scargle, J. D. 1982, ApJ, 263, 835

Strohmayer, T. E., \& Markwardt, C. B. 2010, ATel, 2929, 1

Strohmayer, T. E., Markwardt, C. B., Pereira, D., \& Smith, E. A. 2010, ATel, 2946, 1

Strohmayer, T. E., \& Smith, E. A. 2011, ATel, 3258, 1

Sturner, S. J., \& Dermer, C. D. 1996, ApJ, 465, L31

Taam, R. E. 1981, Ap\&SS, 77, 257

Taam, R. E. 1982, ApJ, 258, 761

Taam, R. E., Woosley, S. E., \& Lamb, D. Q. 1996, ApJ, 459, 271

Tanaka, Y. 1989, in Two Topics in X-Ray Astronomy, Vol. 1: X Ray Binaries. Vol. 2: AGN and the X Ray Background, ed. J. Hunt \& B. Battrick (ESA Special Publication, Vol. 296; Noordwijk: ESA), 3

Truemper, J., Pietsch, W., Reppin, C., et al. 1978, ApJ, 219, L105

Ubertini, P., Bazzano, A., Cocchi, M., et al. 1999, ApJ, 514, L27

van Paradijs, J., Penninx, W., \& Lewin, W. H. G. 1988, MNRAS, 233, 437

Woosley, S. E., Heger, A., Cumming, A., et al. 2004, ApJS, 151, 75

Woosley, S. E., \& Taam, R. E. 1976, Nature, 263, 101

Yu, W., \& van der Klis, M. 2002, ApJ, 567, L67 